Copyright ( 2013 IEEE. Reprinted from "Sun, X. \& Tsang, D.H.K. (2013). Energy-efficient cooperative sensing scheduling for multi-band cognitive radio networks. IEEE Transactions on Wireless Communications, v. 12 (10), 4943-4955."

This material is posted here with permission of the IEEE. Such permission of the IEEE does not in any way imply IEEE endorsement of any of The Hong Kong University of Science and Technology's products or services. Internal or personal use of this material is permitted. However, permission to reprint/republish this material for advertising or promotional purposes or for creating new collective works for resale or redistribution must be obtained from the IEEE by writing to pubs-permissions@ieee.org.

By choosing to view this document, you agree to all provisions of the copyright laws protecting it. 


\title{
Energy-efficient Cooperative Sensing Scheduling for Multi-Band Cognitive Radio Networks
}

\author{
Xiangxia Sun and Danny H. K. Tsang, Fellow, IEEE
}

\begin{abstract}
In this paper, by taking both sensing performance and energy efficiency into consideration, the Cooperative Sensing Scheduling (CSS) problem for multi-band Cognitive Radio Networks (CRNs) is investigated under a practical scenario where both Primary User (PU) channels and Secondary Users (SUs) have heterogeneous characteristics. Unlike many existing works that merely claim that the CSS problem is NP-hard and then turn to heuristic methods, we analyze this problem under a solid discrete-convex framework. After formulating the CSS problem as a nonlinear binary programming problem, we adopt a threestep approach to solve it. In the first step, the number of SUs assigned to sense each PU channel is determined with the $M / M^{\natural}$ convex theory. Based on the results obtained in the first step, we then find the $S U$ assignment using the $L / L^{\natural}$-convex theory in the second step. In the last step, the optimal number of SUs participating in sensing is obtained based on the $\mathrm{SU}$ assignment obtained in step two. By combining these three steps, a complete and efficient $\mathrm{SU}$ assignment scheme is obtained. Numerical results are provided to evaluate the performance of our proposed SU assignment scheme and validate the theoretical analysis.
\end{abstract}

Index Terms-cognitive radio, cooperative sensing scheduling, nonlinear binary programming, energy-efficient, discrete-convex

\section{INTRODUCTION}

The recent popularity of wireless devices such as smart phones and tablets gives rise to a substantially increasing need for higher data rate and more spectrum bandwidth. This trend makes it necessary for the static spectrum allocation policy to evolve to a more advanced and efficient spectrum assignment mechanism. The currently used fixed spectrum allocation method has become a bottleneck which impedes such evolution. The emergence of Cognitive Radio (CR) [1] opens up a new possibility to resolve this problem. CR allows Secondary Users (SUs) to opportunistically access frequency bands which are exclusively allocated to Primary Users (PUs) without introducing harmful interference to the communication process of PUs.

\section{A. Advances in CR and Cooperative Spectrum Sensing}

In a CRN, detecting the presence of PUs is a compulsory procedure before SUs can make use of the PU channels. SUs are required to perform spectrum sensing to determine which portion of PU channels are available before transmitting their data. One single SU, however, either has limited sensing capability (i.e., can only sense a narrow range of PU channels) due to hardware limitations, or produces unreliable sensing results because of various channel conditions such as shadowing and multi-path fading. To meet the stringent sensing performance requirements specified by corresponding regulatory organizations, MAC-layer support is required to improve the accuracy and reliability of spectrum sensing. Cooperative sensing [3], which allows a group of SUs to collaboratively sense one PU channel, is one of such technologies. By allowing multiple SUs to work together on detecting the PU signals from different places and thus exploiting the location diversity, cooperative sensing can improve the sensing performance of the CRN significantly. With its rapid development and recent progresses such as cooperative relay techniques [2], cooperative sensing makes the realization of efficient and reliable CRNs possible.

In addition to advances in cooperative spectrum sensing, CR itself is evolving to support greater data transmission capacity [4] and more flexibility [5] to coexist with other techniques. Especially, recent developments in spectrum regulatory rules, i.e. the release of unused spectrum bands to cognitive devices, have triggered active standardization activities on $\mathrm{CR}$ from various organizations, including IEEE, the International Telecommunication Union (ITU), European Telecommunications Standards Institute (ETIS), and European Association for Standardizing Information and Communication Systems (ECMA) and several standards for CR have already been made (e.g., IEEE 802.22 WRAN and Ecma 392) [6]. With these developments ongoing, it is believed that the implementation and commercialization of $\mathrm{CR}$ are on the horizon.

\section{B. Cooperative Sensing Scheduling (CSS) and Related Works}

CRNs usually co-locate with multiple PU channels in their coverage area. For such multi-band CRNs adopting cooperative sensing techniques, a fundamental problem, which is seldom studied and little understood, is how to appropriately schedule available SUs to sense potential PU channels. It can be further interpreted as "which channel to sense" and "if a channel is sensed, it should be sensed by which SU (or SUs)". This problem is referred to as the Cooperative Sensing Scheduling (CSS) problem. Two steps are needed to determine the appropriate sensing parameters of a CRN. CSS (i.e., the assignment of SUs to sense different PU channels) is the first step. The second step, which is already well studied in [7] [10], is to determine the sensing duration, detection threshold and other sensing parameters of SUs according to different design objectives. This paper focuses on the first step (i.e., the CSS problem).

The fundamental tradeoffs in CSS is as follows: (1) Energyperformance tradeoff: More SUs participating in sensing guarantees a better spectrum discovery outcome but more energy will be consumed. (2) Performance-opportunity tradeoff: Assigning more SUs with high sensing capability to cooperatively 
sense one channel will lead to a better sensing performance, but the CRN cannot fully explore spectrum opportunity since less potential channels are sensed. A desirable SU assignment scheme should achieve a good balance among energy consumption, sensing performance and the exploration of spectrum opportunity.

Research on CSS is far from adequate, especially for "realworld" situations. Only a handful of works can be found [11][18]. In [11], the authors studied the multichannel coordination problem. After modeling it as an integer programming problem, the authors claimed that the problem is NP-hard and then proposed heuristic algorithms to obtain suboptimal solutions. [12] and [13] provided a systematic method to deal with the performance-opportunity tradeoff of the CSS problem mentioned above without considering the energy-performance tradeoff. They assumed homogeneous characteristics on both SUs and PU channels, which is usually not the case in "real world". [14] and [15] are further extension of [12] and [13] with different PU channel detection probability requirements, but the heterogeneous characteristics of SUs are still not considered. [18] provided a comprehensive design which considered both CSS and sensing parameters setting. By assuming the number of SUs sensing all PU channels to be equal and ignoring the energy-performance tradeoff mentioned above, the performance of their proposed SU assignment scheme deteriorated. Similar to [11], authors of [18] also turned to heuristic methods after claiming that the problem is NP-hard.

\section{Contributions}

The contributions of this work are summarized as follows.

- The CSS problem is considered in a more practical setting in which SUs individually have heterogeneous SNRs for different PU channels and PU channels also have heterogeneous characteristics (which include detection probability requirement, channel capacity and stationary idle probability). In real applications, the distances between a PU transmitter and different SUs are often different. The signal propagation environment also differs for a PU transmitter to different SUs. Therefore, SNR for the same primary signal is usually not the same at different SU receivers. On the other hand, SUs with low SNR often require longer spectrum sensing time while providing a less accurate result. What is more, if the SNR is too low, the SU can never detect the PU presence correctly no matter how much time it spends on sensing. Hence, in order to make CRNs function properly, SU assignment scheme for the heterogeneous SNR case needs further study. Especially, when the "OR" rule is adopted, the sensing performance for a certain channel might be dragged down significantly by the inaccurate sensing results of SUs with inferior sensing ability. Therefore, the investigation of how to assign these SUs with different sensing capabilities to sense PU channels with heterogeneous characteristics and requirements is of practical importance.

- We analyze the CSS problem under a solid discreteconvex framework different from many existing works (such as [11] [18]) that merely claim the CSS problem being NP-hard and then proceed to find a heuristic method to solve the problem. For a CRN consisting of SUs with different sensing capabilities and having access to multiple heterogeneous PU channels, the sensing scheduling problem becomes much more challenging than the one considered by existing works [12], [14] and [15]. In this paper, after formulating the CSS problem as a nonlinear binary programming problem, we adopt a threestep approach to solve it. In the first step, the number of SUs assigned to sense each channel is obtained. In the second step, we determine the SU assignment matrix using the $\mathrm{L} / \mathrm{L}^{\mathrm{q}}$-convex theory. In the last step, the optimal number of SUs participating in sensing is found based on the results of the SU assignment scheme obtained in step two. By combining these three steps, a complete and efficient SU assignment scheme is obtained.

- Energy consumption is also considered as one of the CRN design objectives in this paper. By selecting an appropriate number of SUs participating in sensing, we achieve a good balance between the spectrum discovery and the total sensing energy consumption.

\section{Paper Organization}

The rest of this paper is organized as follows. System model is given in section II and the problem formulation is presented in section III. In section IV, we give a preliminary overview on $\mathrm{M} / \mathrm{M}^{\natural}$-convexity, $\mathrm{L} / \mathrm{L}^{\natural}$-convexity and the relationship between them to facilitate further analysis. The background knowledge on polymatroid, greedy algorithm and the relationship between them will also be stated in section IV. We then proceed to analyze the inherent structure of the CSS problem in section V. By dividing this problem into three subproblems, efficient algorithms are proposed to obtain the solution. We also provide numerical results in section VI to evaluate the performance of our proposed SU assignment scheme and validate our theoretical analysis. Finally, we conclude this paper in section VII.

\section{SYSTEM MODEL}

We consider a centralized CRN with $M$ SUs denoted as $\mathcal{M}=\{1,2, \ldots, M\}$ and a Base Station (BS). The BS is in charge of assigning SUs to sense different PU channels and collecting individual sensing results from SUs to make final decisions on the occupancy state of PU channels. SUs can opportunistically access $N$ PU channels, which are denoted as $\mathcal{N}=\{1,2, \ldots, N\}$. Without loss of generality, we assume PU signals are complex PSK modulated and the noise is circularly symmetric complex Gaussian. Let $s_{n}=0\left(s_{n}=1\right)$ denote PU channel $n(n \in \mathcal{N})$ being idle (busy). Also, these PU channels are heterogeneous in terms of channel protection criteria, channel idle probabilities and channel capacities. Throughout this paper, we assume that the SUs are equipped with single radio interface, and therefore can only sense or access one PU channel at a time. We assume the CRN works in a slotted frame structure [10] and the frame length is denoted as $T$. Each frame is divided into three parts, namely, sensing 
duration $\tau$, scheduling and results fusion duration $\eta$, and data transmission duration $T-\tau-\eta$. Since the design of controlling signal is beyond the scope of this paper, we do not make any specific assumption on this. The controlling signal can be sent and received through either a dedicated control channel or a specially designed scheme without common control channels [17].

In contrast to previous works [12], [13], [14] and [15], the signal to noise ration (SNR) at each SU for different PU channels is heterogeneous. Denote $\Gamma=\left\{\gamma_{m, n}\right\}$ as the SNR matrix, where SNR of SU $m(m \in \mathcal{M})$ for PU Channel $n(n \in \mathcal{N})$ is denoted as $\gamma_{m, n}$. In the sensing duration, each SU is either assigned to sense one PU channel or not to sense any channel. We assume energy detection [19] is adopted by all SUs during the sensing process. Spectrum sensing performance of each SU is measured by the detection probability $p_{d}$ and the false alarm probability $p_{f}$, which are defined as follows

$$
\begin{aligned}
& p_{d} \triangleq \operatorname{Pr}\left\{\text { decide } \mathcal{H}_{1} \mid \mathcal{H}_{1} \text { is true }\right\}, \\
& p_{f} \triangleq \operatorname{Pr}\left\{\text { decide } \mathcal{H}_{1} \mid \mathcal{H}_{0} \text { is true }\right\},
\end{aligned}
$$

where $\mathcal{H}_{1}$ and $\mathcal{H}_{0}$ denote the hypotheses that the PU is present and absent, respectively.

Suppose that the CRN adopts cooperative spectrum sensing. All SUs report their one bit sensing results to the BS after individually performing spectrum sensing based on energy detection. The BS combines the collected results using "OR" rule $^{1}$ to obtain the final decision on the occupancy state of each PU channel. Denote $X=\left\{x_{m, n}\right\}$ as the SU assignment matrix, and $x_{m, n}=1\left(x_{m, n}=0\right)$ means that SU $m$ is (not) assigned to sense PU channel $n$. In order to facilitate further analysis, the column vector expression of the assignment matrix denoted as $X=\left[\mathbf{x}_{1}, \mathbf{x}_{2}, \cdots, \mathbf{x}_{N}\right]$ is also introduced, where $\mathbf{x}_{n}=\left[x_{1, n}, x_{2, n}, \cdots, x_{M, n}\right]^{T}$. Let $a_{n}=\sum_{m} x_{m, n}$ represent the number of SUs assigned to sense PU channel $n$. Denote 0 (1) as a vector where all elements are 0 (1). The sensing performance in terms of the probability of detection and false alarm for channel $n$ at the BS when $\mathbf{x}_{n} \neq \mathbf{0}$ can be expressed as

$$
\begin{aligned}
& P_{d, n}=1-\prod_{m}\left(1-p_{d, m, n}\right)^{x_{m, n}}, \\
& P_{f, n}=1-\prod_{m}\left(1-p_{f, m, n}\right)^{x_{m, n}},
\end{aligned}
$$

where $p_{d, m, n}$ and $p_{f, m, n}$ denote the individual detection probability and false alarm probability of SU $m$ for PU channel $n$, respectively. We assume that the individual detection probability for all the SUs sensing the same channel are the same. Actually, this assumption can be fulfilled by adjusting the individual energy detection threshold. With this assumption, the detection probability for PU channel $n$ at the BS can be expressed as

$$
P_{d, n}=1-\left[1-p_{d, n}\right]^{a_{n}},
$$

\footnotetext{
${ }^{1}$ The results in this draft can be easily extended to AND rule since we only need to change the formula of detection probability and false alarm probability given in equation (2) to the following form: $P_{d, n}=\prod_{m} p_{d, m, n}{ }^{x_{m, n}}$, $P_{f, n}=\prod_{m} p_{f, m, n}{ }^{x_{m, n}}$. All the theorems, lemmas and propositions are still applicable.
}

where $p_{d, n}$ is the individual detection probability of $\mathrm{SU}$ for channel $n$. The relationship between individual detection and false alarm probabilities for each SU is given by [10]

$$
p_{f, m, n}=Q\left(\sqrt{2 \gamma_{m, n}+1} Q^{-1}\left(p_{d, n}\right)+\sqrt{\tau f_{s}} \gamma_{m, n}\right),
$$

where $f_{s}$ denotes the sampling rate, $Q(\cdot)$ represents the Qfunction (i.e., the tail probability of the standard Gaussian distribution) and $Q^{-1}(\cdot)$ is the inverse function of $Q(\cdot)$. Notice that the individual false alarm probability is still heterogeneous for different SUs sensing the same channel as shown in equation (4). In a scenario where Raleigh fading is considered, the above equation (4) should be replaced by the following one [16]

$$
p_{f, m, n}=Q\left(\frac{2\left|h_{m, n}\right|^{2} \gamma_{m, n} \varepsilon \sqrt{\tau f_{s}}}{\sigma_{u}^{2}\left(2\left|h_{m, n}\right|^{2} \gamma_{m, n}+1\right)}+Q^{-1}\left(p_{d, m, n}\right)\right),
$$

where $h_{m, n}$ is the fading coefficient, $\varepsilon$ is the detection threshold and $\sigma_{u}^{2}$ is the noise power. All the theoretical analyses in this paper are still applicable in this case.

\section{PROBLEM Formulation}

Two essential tradeoffs lie in the CSS problem. The first tradeoff addresses the conflict between sensing energy consumption and sensing performance. Letting more SUs participate in the spectrum sensing process will lead to a better performance at the cost of consuming more energy. The second tradeoff lies between sensing accuracy and spectrum opportunity exploration. If many well performed SUs are assigned to sense a single channel cooperatively, the sensing accuracy of that channel is higher, but fewer channels are sensed within duration $\tau$ consequently. On the other hand, if the SUs are scattered sparsely across the channels, sensing accuracy will deteriorate although the number of channels sensed increases. To tackle these tradeoffs, we need to find a proper assignment of SUs to sense PU channels such that a good balance among sensing energy consumption, spectrum opportunity exploration and sensing accuracy is achieved.

We notice that the number of SUs assigned to sense channel $n$ (i.e., $a_{n}$ ) might be 0 , namely, it is possible that channel $n$ is not sensed. However, function $P_{f, n}\left(\mathbf{x}_{n}\right)$ is not defined when $\mathbf{x}_{n}=\mathbf{0}$. To address this problem, the false alarm probability function is extended as follows

$$
\bar{P}_{f, n}\left(\mathbf{x}_{n}\right)=\left\{\begin{array}{ccc}
1 & \text { for } & \mathbf{x}_{n}=\mathbf{0} \\
P_{f, n}\left(\mathbf{x}_{n}\right) & \text { for } & \mathbf{x}_{n} \neq \mathbf{0} .
\end{array}\right.
$$

The above extension of the false alarm probability function $\bar{P}_{f, n}\left(\mathbf{x}_{n}\right)$ defines the false alarm probability as 1 when $\mathbf{x}_{n}=$ $\mathbf{0}$. The physical meaning of this extension is as follows. If a channel is not sensed and thus could not be utilized by SUs, it will not give any gain or loss to the aggregated data rate, i.e., channels that are not sensed will neither be rewarded nor punished in terms of aggregated data rate. It will be shown later that this extension will greatly simplify the problem by adding some nice properties to it.

During the sensing period, SUs consume energy at two different power levels, namely, sensing power $\phi_{s}$ and idling power $\phi_{e}$. We assume that all SUs stay quiet and listen for the PU signals during the sensing duration, i.e., SUs stop packet 
transmission and listen to the PU channels. Suppose that SUs participating in sensing consume energy at power level $\phi_{s}$ and SUs not participating in sensing consume energy at power level $\phi_{e}$. The total energy consumption in the sensing duration is given by

$$
E(\kappa)=\tau \phi_{s} \kappa+\tau \phi_{e}(M-\kappa),
$$

where $\kappa$ is the number of SUs participating in sensing.

Denote $X(\kappa)=\left[\mathbf{x}_{1}(\kappa), \mathbf{x}_{2}(\kappa), \cdots, \mathbf{x}_{N}(\kappa)\right]$ as the $\mathrm{SU}$ assignment matrix corresponding to the variable $\kappa$. The $n$ th column sum of matrix $X(\kappa)$ is represented by $a_{n}(\kappa)$. Let $C_{0, n}$ and $C_{1, n}$ denote the capacity of channel $n$ when SUs operate in the scenario where PU of channel $n$ is absent and present, respectively. The aggregated expected data rate, which is represented by $R_{G}(X(\kappa))$, can be expressed as

$$
\begin{aligned}
& R_{G}(X(\kappa))=\sum_{n}\left\{C_{0, n}\left[1-\bar{P}_{f, n}\left(\mathbf{x}_{n}(\kappa)\right)\right] P_{n}\left(\mathcal{H}_{0}\right)+\right. \\
& \left.C_{1, n}\left[1-P_{d, n}\left(\mathbf{x}_{n}(\kappa)\right)\right] P_{n}\left(\mathcal{H}_{1}\right)\right\},
\end{aligned}
$$

where $P_{n}\left(\mathcal{H}_{0}\right)$ and $P_{n}\left(\mathcal{H}_{1}\right)$ denote the stationary probability that channel $n$ is idle and busy, respectively.

According to [10], $C_{0, n}>>C_{1, n}$, and thus the first term on the right hand side of (8) dominates. Besides, only data transmitted when the PU signal is absent, which is represented by the first term on the right hand side of (8), are meaningful and should be encouraged. Hence, we can rewrite the aggregated expected data rate as

$$
\widetilde{R}_{G}(X(\kappa))=\sum_{n} C_{0, n}\left[1-\bar{P}_{f, n}\left(\mathbf{x}_{n}(\kappa)\right)\right] P_{n}\left(\mathcal{H}_{0}\right) .
$$

We have two objective functions: energy consumption objective function (7) and aggregated data rate objective function (9). Therefore, the problem is a multiple objective optimization problem in nature. Weighted global criteria method [20] is a commonly used technique to solve multiple objective optimization problems. All objective functions are combined to form a single global function under this technique. Using this technique, we add energy consumption and aggregated expected data rate together to form a weighted global criteria function, which is called utility function hereafter. The utility function ${ }^{2}$ combining the aggregated expected data rate and energy consumption can be given by

$$
U_{G}(X(\kappa), \kappa)=w_{r} \widetilde{R}_{G}(X(\kappa))-w_{e} E(\kappa),
$$

where $w_{r} \geq 0$ and $w_{e} \geq 0$ are two weighting factors for the aggregated data rate and energy consumption, respectively.

In order to protect the quality of service of PUs, the achieved detection probability of sensed channels should not be smaller than the required threshold. Denote $\lambda_{n}$ as detection probability requirement imposed on channel $n$. Therefore, the

\footnotetext{
${ }^{2}$ Throughout the paper, utility function refers to function (10).
}

CSS problem can be formulated as follows

$$
\begin{aligned}
\text { (GCSS }): & \max _{X(\kappa), \kappa} U_{G}(X(\kappa), \kappa) \\
\text { s.t. } \quad & \sum_{n=1}^{N} \sum_{m=1}^{M} x_{m, n}=\kappa \leq M, \\
& \sum_{n=1}^{N} x_{m, n} \leq 1, \\
& x_{m, n} \in\{0,1\}, \\
& P_{d, n}(X(\kappa)) \geq \lambda_{n}, \forall n \in \mathcal{N} .
\end{aligned}
$$

Constraint (11) means that not all SUs available in the system are required to participate in sensing. Constraint (12) captures our system assumption that SUs are equipped with single interface, i.e., each SU can sense one PU channel at most during the sensing period. Problem (GCSS) is a nonlinear binary programming problem, and the complexity to solve this kind of problem is usually high. Later, we will explore the inherent structure of problem (GCSS) and find some useful properties which are conducive to solving this problem efficiently.

\section{Preliminaries}

To facilitate further analysis, we now provide the preliminary knowledge on $\mathrm{M} / \mathrm{M}^{\natural}$-convexity, $\mathrm{L} / \mathrm{L}^{\natural}$-convexity and the relationship between them. The background knowledge on polymatroid, greedy algorithm and the relationship between them will also be given in this section.

\section{A. $M / M^{\natural}$-convex Functions}

In this subsection, we first introduce the following definitions and theorems. Interested readers can refer to [21] for more details.

Definition 1. For a vector $\mathbf{a}=\left\{a_{v} \mid v \in V\right\}$, the positive and negative support of this vector is defined as

$$
\operatorname{supp}^{+}(\mathbf{a})=\left\{v \mid a_{v}>0\right\}, \operatorname{supp}^{-}(\mathbf{a})=\left\{v \mid a_{v}<0\right\} .
$$

Definition 2. A function $f: Z^{V} \rightarrow R \cup\{+\infty\}^{3}$ with $\operatorname{dom} f \neq$ $\emptyset$ is an M-convex function if it satisfies the following exchange axiom $(\boldsymbol{M}-\boldsymbol{E X C})$ :

(M-EXC) For $\mathbf{a}, \mathbf{b} \in \operatorname{dom} f$ and $u \in \operatorname{supp}^{+}(\mathbf{a}-\mathbf{b})$, there exists $v \in \operatorname{supp}^{-}(\mathbf{a}-\mathbf{b})$ such that

$$
f(\mathbf{a})+f(\mathbf{b}) \geq f\left(\mathbf{a}-\boldsymbol{\chi}_{u}+\boldsymbol{\chi}_{v}\right)+f\left(\mathbf{b}+\boldsymbol{\chi}_{u}-\boldsymbol{\chi}_{v}\right),
$$

where $\chi_{u}$ and $\chi_{v}$ are unit vectors.

One possible interpretation of Definition 2 is as follows: two points stay "far away" from each other have a larger total value than two points which are relatively "close" to each other. The exchange axiom (M-EXC) gives a specific fashion for two points approaching each other. An illustration of Definition 2 and the exchange axiom (M-EXC) for a two dimensional case is given in Fig. 1. Note that we show only the coordinates

\footnotetext{
${ }^{3}$ The notation $Z^{V}$ means the integer vector space with coordinates indexed by the elements of $V$.
} 
of the different points in space without showing the actual function values.

Definition 3. A function $g$ is said to be $M$-concave if $-g$ is M-convex.

Theorem 1. A separable convex (concave) function

$$
f(\mathbf{a})=\sum_{n=1}^{N} f_{n}\left(a_{n}\right) \quad(\mathbf{a} \in \operatorname{dom} f)
$$

with univariate discrete-convex (concave) functions $f_{n}\left(a_{n}\right)$ is M-convex (M-concave).

Definition 4. A function $f: Z^{V} \rightarrow R \cup\{+\infty\}$ with domf $\neq \emptyset$ is $M^{\natural}$-convex ( $M$ natural convex) if and only if $f$ satisfies the following exchange axiom $\left(\boldsymbol{M}^{\natural}-\boldsymbol{E X C}\right)$ :

$\left(\boldsymbol{M}^{\natural}-\boldsymbol{E X C}\right)$ For $\mathbf{a}, \mathbf{b} \in \operatorname{dom} f$,

$\max _{u \in \operatorname{supp}^{+}(\mathbf{a}-\mathbf{b})} \min _{v \in \operatorname{supp}^{-}(\mathbf{a}-\mathbf{b}) \cup\{0\}}[\delta f(\mathbf{a}, v, u)+\delta f(\mathbf{b}, u, v)] \leq 0$,

where $\delta f(\mathbf{a}, v, u)=f\left(\mathbf{a}+\chi_{v}-\chi_{u}\right)-f(\mathbf{a}), \delta f(\mathbf{b}, u, v)=$ $f\left(\mathbf{b}+\chi_{u}-\chi_{v}\right)-f(\mathbf{b})$ and $\chi_{0}=\mathbf{0}$.

Corollary 1. A univariate function $f: Z \rightarrow R$ is $M^{\natural}$-convex if and only if $f$ satisfies the following condition:

$$
f(a)+f(b) \geq f(a-1)+f(b+1),
$$

where $a, b \in \operatorname{dom} f$.

\section{B. $L / L^{\natural}$-convex Functions}

In this subsection, definitions of $\mathrm{L} / \mathrm{L}^{\natural}$-convex functions will be introduced and more detailed information can be found in [21] and [24]. The definitions of $L / L^{\natural}$-convex and $\mathrm{L} / \mathrm{L}^{\natural}$-concave functions are strongly related to the concept of submodular and supermodular, respectively. Hence, we first introduce the following definitions.

Definition 5. Let $\mathbf{x} \wedge \mathbf{y}=\min \{\mathbf{x}, \mathbf{y}\}$ and $\mathbf{x} \vee \mathbf{y}=\max \{\mathbf{x}, \mathbf{y}\}$ represent the element-wise minimum and maximum of vector $\mathbf{x}$ and $\mathbf{y}$, respectively. A function $f: Z^{V} \rightarrow R \cup\{+\infty\}$ is

(1) a submodular function if and only if when

$$
f(\mathbf{x})+f(\mathbf{y}) \geq f(\mathbf{x} \wedge \mathbf{y})+f(\mathbf{x} \vee \mathbf{y})
$$

(2) a supermodular function if and only if when

$$
f(\mathbf{x})+f(\mathbf{y}) \leq f(\mathbf{x} \wedge \mathbf{y})+f(\mathbf{x} \vee \mathbf{y}) .
$$

Based on the definitions given above, the concept of L-convex and $\mathrm{L}^{\natural}$-convex are given as follows [21].

Definition 6. A function $f: Z^{V} \rightarrow R \cup\{+\infty\}$ with $\operatorname{dom} f \neq \emptyset$ is said to be L-convex if it satisfies

(1) $f$ is submodular,

(2) $\exists r \in R$ such that $f(\mathbf{x}+\mathbf{1})=f(\mathbf{x})+r$.

Definition 7. A function $f: Z^{V} \rightarrow R \cup\{+\infty\}$ is called $L^{\natural}$-convex ( $L$ natural convex) if it satisfies the following generalized submodularity (or translation submodularity)

$$
f(\mathbf{x})+f(\mathbf{y}) \geq f((\mathbf{x}-\rho \mathbf{1}) \wedge \mathbf{y})+f(\mathbf{x} \vee(\mathbf{y}+\rho \mathbf{1})),
$$

where $\rho$ is a scalar.

Fig. 2 graphically illustrates the definitions of $\mathrm{L} / \mathrm{L}^{\natural}$-convex functions. Again, we show only the coordinates of the different points in space without showing the actual function values. For $\mathrm{L}^{\natural}$-convex functions, the following theorem holds according to [24].

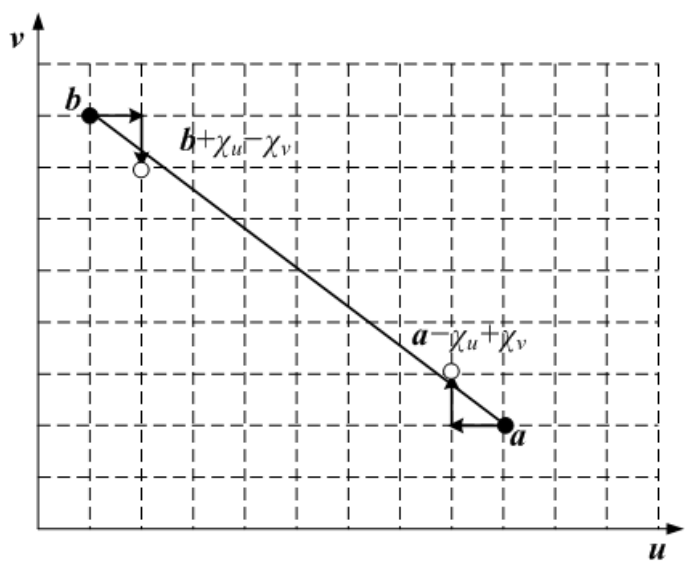

Fig. 1. Illustration for M-convex

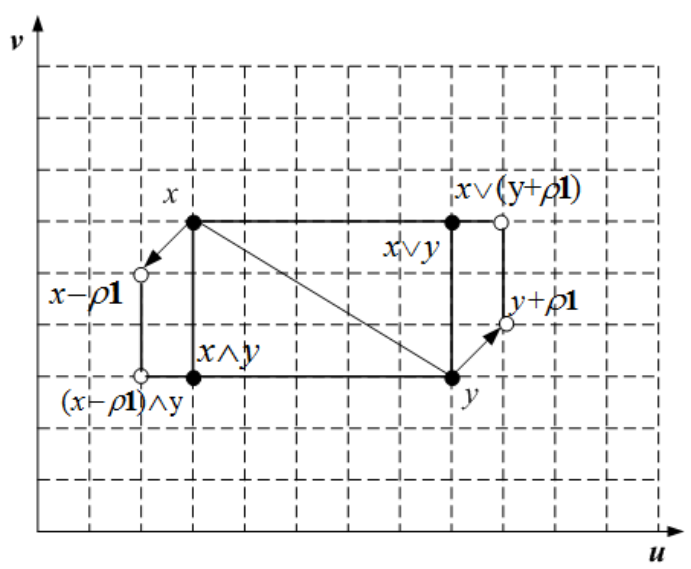

Fig. 2. Illustration for $\mathrm{L}^{\natural} \mathrm{L}^{\natural}-\operatorname{convex}(\rho=1)$

Theorem 2. Let $f_{1}, f_{2} \in \mathcal{L}^{\natural}[Z \rightarrow R]$ be $L^{\natural}$-convex functions, the sum $f_{1}+f_{2}$ is still $L^{\natural}$-convex provided $\operatorname{dom}\left(f_{1}+f_{2}\right) \neq \emptyset$, where $\mathcal{L}^{\natural}[Z \rightarrow R]$ denotes the set of $L^{\natural}$-convex functions.

Particularly, for a function with binary variables, we have the following theorem and corollary [24].

Theorem 3. A function $f$ with $\operatorname{dom} f \subseteq\{0,1\}^{V}$ is $L^{\natural}$-convex if and only if it is submodular.

Corollary 2. A function $f$ with $\operatorname{dom} f \subseteq\{0,1\}^{V}$ is $L^{\natural}$ concave if and only if it is supermodular.

\section{Relationship among Discrete-convex Functions}

In this subsection, we clarify the relationship between $\mathrm{L} / \mathrm{L}^{\natural}$ convex and $\mathrm{M} / \mathrm{M}^{\natural}$-convex functions which have been introduced in the previous two subsections. $\mathbf{M}^{\natural}$-convex functions 
are conceptually equivalent to M-convex functions since an $\mathrm{M}^{\natural}$-convex function can be derived from the projection of the corresponding M-convex function to a lower dimension. However, the class of $\mathrm{M}^{\natural}$-convex functions contains that of $\mathrm{M}$ convex functions. A simple example is as follows: $\mathbf{M}^{\natural}$-convex functions allow $\chi_{v}=\mathbf{0}$ while $\mathrm{M}$-convex functions do not. Lconvex functions are special cases of $\mathrm{L}^{\natural}$-convex functions for $\rho=0$. Both $\mathrm{L}^{\natural}$-convex and $\mathrm{M}^{\natural}$-convex functions are integrally convex functions [24] as shown in the following lemma.

Lemma 1. A function $f: Z^{V} \rightarrow R \cup\{+\infty\}$ is integrally convex (concave) if it is $L^{\natural}$-convex ( $L^{\natural}$-concave) or $M^{\natural}$-convex ( $M^{\natural}$-concave).

\section{Polymatroid and Greedy Algorithm}

In this subsection, the definition and properties of polymatroid will be given. Generally speaking, a polymatroid is a polytope associated with a submodular function. Here we give a detailed definition [22]. Note that the interpretation of $T$ and $a_{i}$ should be applied only within the context of Definition 8 .

Definition 8. For a set $V$, it's power set or subset is denoted as $2^{V}=\{S: S \subseteq V\}$. $F$ denotes a set function $F$ on $2^{V}$ which satisfies the following conditions:

(1) $F$ is nonnegative: $F(S) \geq 0$,

(2) $F$ is nondecreasing: $F(S) \leq F(T)$ if $S \subseteq T \subseteq V$,

(3) $F$ is submodular: $F(S)+F(T) \geq F(S \cup T)+F(S \cap T)$. $G=\mathcal{G}\left(2^{V}, F\right)=\left\{\mathbf{g} \in \mathbf{N}^{V}: \mathbf{a}(S) \leq F(S)\right\}$ is called $a$ Polymatroid, where $\mathrm{g}(S)=\sum_{i \in S} a_{i}$ and $\mathrm{N}^{V}$ denotes a vector with $i$-th unit basis as $e^{i}(i \in V)$. $V$ is the ground set and $F$ is the rank function of polymatroid $G$.

The greedy algorithm which is also refereed to as marginal allocation algorithm assigns the available resources sequentially unit by unit to the activities. In each step, the resource unit is assigned to the activity which gives the largest increase to the objective function ( i.e., gives the largest benefit to the whole system) if one additional unit or allotment is assigned to that activity. The assignment procedure ends either when the resources are finished or when no increase can be found. Greedy algorithm is very efficient and easy to implement. However, the optimality of greedy algorithm is not guaranteed generally, but in the special case described in the following Lemma 2, the greedy algorithm indeed gives an optimal solution [23]. With the concept of polymatroid and greedy algorithm presented, we now give the relationship between them in the following lemma.

Lemma 2. Greedy or marginal allocation procedure will give an optimal solution to the discrete optimization problem if the objective function is concave and the underlying topology of the feasible region is a polymatroid.

\section{Problem Analysis}

In this section, the following three steps are taken sequentially to analyze problem (GCSS):

Step S1: For a given $\kappa$, find the optimal number of SUs assigned to sense channel $n$, i.e., $a_{n}^{*}(\kappa)$, for each PU channel $n \in \mathcal{N}$.
Step S2: Based on $a_{n}^{*}(\kappa)$ obtained in Step S1, find the assignment of SUs to sense different PU channels, i.e., $X$ given $\mathbf{a}^{*}(\kappa)$.

Step S3: Given the SU assignment (i.e., $X$ ) obtained in Step $\mathrm{S} 2$, find the number of SUs participating in sensing, i.e., $\kappa$.

Each subsection in the remaining part of this section will carry out one individual step. Note that the order of these three steps given above is only for theoretical analysis, and in algorithm design and implementation, these three steps are taken iteratively to solve problem (GCSS).

\section{A. Find $a_{n}^{*}(\kappa)$ for a Fixed $\kappa$}

Before proceeding to the next step of solving this problem, we should first find $a_{n}$ for each channel $n \in \mathcal{N}$. Recall that $a_{n}=\sum_{m} x_{m, n}$ is the number of SUs assigned to sense channel $n$. In this subsection, we approximate the SNR by the average SNR for each channel. Denote the average SNR for channel $n$ as $\bar{\gamma}_{n}$, i.e, $\bar{\gamma}_{n}=\frac{1}{M} \sum_{m} \gamma_{m, n}$. The optimal $a_{n}^{*}$ will be found in the following analysis based on this approximation. Note that the SUs' sensing capabilities are approximated to be homogeneous in this subsection. Therefore, the individual false alarm probability can be expressed as

$$
p_{f, n}=Q\left(\sqrt{2 \bar{\gamma}_{n}+1} Q^{-1}\left(p_{d, n}\right)+\sqrt{\tau f_{s}} \bar{\gamma}_{n}\right) .
$$

Consequently, the extended false alarm probability for channel $n$ at the $\mathrm{BS}$ is given by

$$
\bar{P}_{f, n}\left(a_{n}\right)=\left\{\begin{array}{lll}
1 & \text { for } & a_{n}=0 \\
1-\left[1-p_{f, n}\right]^{a_{n}} & \text { for } & a_{n} \neq 0 .
\end{array}\right.
$$

For fixed $\kappa$, the energy consumption (i.e., second) term of utility function (10) becomes a constant, therefore, we can ignore that term in this subsection and subsection V-B. We will deal with that term in subsection V-C. Denote $U(\mathbf{a}(\kappa))$ as the first term of utility function (10) under the approximation mentioned above when $\kappa$ is fixed, and $U(\mathbf{a}(\kappa))$ can be expressed as

$$
U(\mathbf{a}(\kappa))=\sum_{n} \alpha_{n}\left[1-\bar{P}_{f, n}\left(a_{n}\right)\right]
$$

where $\alpha_{n}=w_{r} C_{0, n} P_{n}\left(\mathcal{H}_{0}\right)$. Hence, subproblem (CSS1) is given as follows

$$
\begin{aligned}
\text { (CSS1) : } & \max _{\mathbf{a}} U(\mathbf{a}(\kappa)) \\
\text { s.t. } & \sum_{n=1}^{N} a_{n}=\kappa \leq M, \\
& P_{d, n}\left(a_{n}\right) \geq \lambda_{n}, \forall n \in \mathcal{N} .
\end{aligned}
$$

Subproblem (CSS1) is a nonlinear integer programming problem, and the complexity to solve this kind of problem is usually high. In the remaining part of this subsection, we will explore the inherent characteristics of subproblem (CSS1), and we expect to find some useful properties which are conducive to solving this problem efficiently.

The following lemma [13] [15] provides us with further insight to solve this problem. Here we drop the subscript $n$ and use $P_{f}$ and $p_{f}$ to represent the overall and individual false alarm probabilities, respectively. 
Lemma 3. Let $m(m \in[1, \infty))$ be a continuous variable representing the number of SUs assigned to sense a channel. $P_{f}(m)$ is decreasing and convex if the following condition holds

$$
\begin{array}{r}
{\left[\ln \left(1-p_{f}(m)\right)-\frac{m}{1-p_{f}(m)} \nabla p_{f}(m)\right]^{2}} \\
<\frac{2 \nabla p_{f}(m)-m \nabla^{2} p_{f}(m)}{1-p_{f}(m)}-\left[\frac{\sqrt{m} \nabla p_{f}(m)}{1-p_{f}(m)}\right]^{2} .
\end{array}
$$

By extensive simulation, we find that condition (28) holds for most practical systems [13]. Without loss of generality, we assume condition (28) holds throughout this paper. According to Lemma 3, $P_{f}(m)$ is decreasing and convex when condition (28) holds and variable $m$ is continuous. However, in subproblem (CSS1), the variables are integer numbers. Also, the concept of convex function becomes much more complicated in discrete case. Therefore, we need to analyze subproblem (CSS1) in the framework of $\mathrm{M} / \mathrm{M}^{\natural}$-convexity which is different from traditional continuous convex analysis.

Lemma 4. Let $a_{n} \in\{0,1, \ldots, \kappa\}$ be the number of $S U$ s assigned to sense channel $n$, then $\bar{P}_{f, n}\left(a_{n}\right)$ is an $M^{\natural}$-convex function if the following two conditions hold

(1) $\bar{P}_{f, n}(1) \leq 0.5+\bar{P}_{f, n}(2) / 2$,

(2) Condition (28) holds for $a_{n} \in[1, \infty)$.

Proof: Case 1: $a_{n}^{\prime}, a_{n}^{\prime \prime} \in\{1, \ldots, \kappa\}$

Note that $a_{n}^{\prime}$ and $a_{n}^{\prime \prime}$ are scalars with integer value. According to Lemma $3, P_{f}(m)$ is decreasing and convex. Hence, $\bar{P}_{f}(m)$, the extended false alarm probability, is decreasing and convex for any $m \in[1, \infty)$. Therefore, for any $a_{n}^{\prime} \leq a_{n}^{\prime \prime}\left(a_{n}^{\prime}, a_{n}^{\prime \prime} \in\right.$ $\{1, \ldots, \kappa\})$, we have

$$
\bar{P}_{f, n}\left(a_{n}^{\prime}\right)-\bar{P}_{f, n}\left(a_{n}^{\prime}+1\right) \geq \bar{P}_{f, n}\left(a_{n}^{\prime \prime}-1\right)-\bar{P}_{f, n}\left(a_{n}^{\prime \prime}\right) .
$$

Rearranging equation (29) gives the following equation

$$
\bar{P}_{f, n}\left(a_{n}^{\prime \prime}\right)+\bar{P}_{f, n}\left(a_{n}^{\prime}\right) \geq \bar{P}_{f, n}\left(a_{n}^{\prime \prime}-1\right)+\bar{P}_{f, n}\left(a_{n}^{\prime}+1\right) .
$$

According to Corollary 1, equation (30) reveals that $\bar{P}_{f, n}\left(a_{n}\right)$ is an $\mathrm{M}^{\natural}$-convex function.

Case 2: $a_{n}^{\prime}, a_{n}^{\prime \prime} \in\{0,1, \ldots, \kappa\}$

Similarly, we assume $a_{n}^{\prime}<a_{n}^{\prime \prime}\left(a_{n}^{\prime}, a_{n}^{\prime \prime} \in\{0, \ldots, \kappa\}\right)$. Since the scenario in which $a_{n}^{\prime}, a_{n}^{\prime \prime} \in\{1, \ldots, \kappa\}$ has already been proven in the above Case 1 and $a_{n}^{\prime}<a_{n}^{\prime \prime}$, we only need to prove that equation (19) of Corollary 1 holds for $\bar{P}_{f, n}\left(a_{n}\right)$ when $a_{n}^{\prime}=0$ and $a_{n}^{\prime \prime} \in\{1, \ldots, \kappa\}$.

- $a_{n}^{\prime}=0$ and $a_{n}^{\prime \prime}=1$

In this case, we have

$$
\bar{P}_{f, n}\left(a_{n}^{\prime \prime}\right)+\bar{P}_{f, n}\left(a_{n}^{\prime}\right)=\bar{P}_{f, n}\left(a_{n}^{\prime \prime}-1\right)+\bar{P}_{f, n}\left(a_{n}^{\prime}+1\right) .
$$

This indicates that equation (19) holds for $\bar{P}_{f, n}\left(a_{n}\right)$ when $a_{n}^{\prime}=0$ and $a_{n}^{\prime \prime}=1$.

- $a_{n}^{\prime}=0$ and $a_{n}^{\prime \prime}>1$

Since $\bar{P}_{f, n}(1) \leq 0.5+\bar{P}_{f, n}(2) / 2$, we have $1-\bar{P}_{f, n}(1) \geq$ $\bar{P}_{f, n}(1)-\bar{P}_{f, n}(2)$. From Case 1 , we obtain $\bar{P}_{f, n}(1)-$ $\bar{P}_{f, n}(2) \geq \bar{P}_{f, n}\left(a_{n}^{\prime \prime}-1\right)-\bar{P}_{f, n}\left(a_{n}^{\prime \prime}\right)$. Hence, we have $1-\bar{P}_{f, n}(1) \geq \bar{P}_{f, n}\left(a_{n}^{\prime \prime}-1\right)-\bar{P}_{f, n}\left(a_{n}^{\prime \prime}\right)$. Thus we obtain $\bar{P}_{f, n}(0)-\bar{P}_{f, n}(1) \geq \bar{P}_{f, n}\left(a_{n}^{\prime \prime}-1\right)-\bar{P}_{f, n}\left(a_{n}^{\prime \prime}\right)$. The above

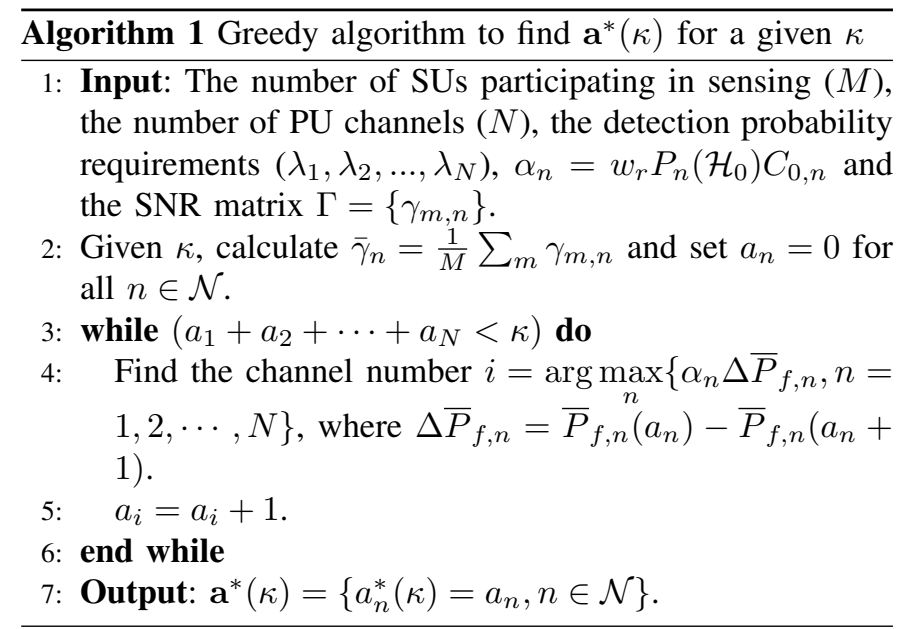

equation equals to the following equation

$$
\bar{P}_{f, n}\left(a_{n}^{\prime \prime}\right)+\bar{P}_{f, n}\left(a_{n}^{\prime}\right) \geq \bar{P}_{f, n}\left(a_{n}^{\prime \prime}-1\right)+\bar{P}_{f, n}\left(a_{n}^{\prime}+1\right)
$$

This indicates that equation (19) holds for $\bar{P}_{f, n}\left(a_{n}\right)$ when $a_{n}^{\prime}=0$ and $a_{n}^{\prime \prime} \geq 2$.

The above analysis and deduction show that equation (19) holds for $\bar{P}_{f, n}\left(a_{n}\right)$ when $a_{n}^{\prime}, a_{n}^{\prime \prime} \in\{0,1, \ldots, \kappa\}$. This proves that $\bar{P}_{f, n}\left(a_{n}\right)$ is an $\mathrm{M}^{\natural}$-convex function for $a_{n} \in\{0,1, \ldots, \kappa\}$.

Throughout this paper, we assume that condition (1) in Lemma 4 holds for every channel $n \in \mathcal{N}$. Actually, this assumption can be easily met in practical CRNs since many standards (e.g., [25] and [26]) explicitly specify that the false alarm probability should be smaller than 0.5 . This requirement of false alarm probability guarantees that condition (1) in Lemma 4 is satisfied. Based on Lemma 4, we have the following proposition.

Proposition 1. The objective function in subproblem (CSS1), i.e., $U(\mathbf{a}(\kappa))$, is M-concave.

Proof: Let $g_{n}\left(a_{n}\right)=\alpha_{n}\left[1-\bar{P}_{f, n}\left(a_{n}\right)\right]$. Since $\alpha_{n}$ is a positive scalar for all channels, $g_{n}\left(a_{n}\right)$ is M-concave according to Lemma 4 and Definition 3. By referring to equation (25), we have $U(\mathbf{a}(\kappa))=\sum_{n} g_{n}\left(a_{n}\right)$. Function $U(\mathbf{a}(\kappa))$ consists of separable M-concave functions $g_{n}$. According to Theorem $1, U(\mathbf{a}(\kappa))$ is M-concave.

Similar to [10], we can easily prove that the optimal solution of subproblem (CSS1) is achieved at equality in constraint (27) (see Appendix A for the detailed proof). According to the relationship between $P_{d, n}$ and $P_{f, n}$ given by equation (50) in Appendix A, we can always make the equality in constraint (27) hold. Therefore, the feasible region of subproblem (CSS1) is only determined by the linear constraint (26). According to Definition 8, the underlying mathematical topology of constraint (26) is a polymatroid. Detailed proof is given in Appendix B. Therefore, based on Lemma 2 and Proposition 1, we can conclude that greedy algorithm gives the optimal solution to subproblem (CSS1). Hence, the following greedy Algorithm 1 can be used to find the optimal value of $a_{n}(\forall n \in \mathcal{N})$ when $\kappa$ SUs participating in sensing. 
Algorithm 1 assigns the given $\kappa$ SUs to the PU channels one by one in a greedy manner. The vector $\mathbf{a}^{*}(\kappa)$ denotes the optimal number of SUs assigned to each channel for a given $\kappa$ according to Algorithm 1. Later, we will use $\mathbf{a}^{*}(\kappa)$ and $\mathbf{a}^{*}$ interchangeably to denote the result obtained from Algorithm 1. In each iteration, Algorithm 1 searches for the channel that gives the largest improvement to the objective function of subproblem (CSS1) and assigns the SU to that channel. The physical meaning of lines 4 and 5 is as follows: when one additional SU becomes available, the CRN will compare the improvement of false alarm probability for each channel if this $\mathrm{SU}$ is assigned to sense that channel. This SU will then be assigned to the channel which gives the largest improvement. Computational complexity of this algorithm is $O(\kappa N)$, which is linear in both $\kappa$ and $N$.

\section{$B$. Find the SU Assignment $X$ for a Given $\mathbf{a}^{*}$}

In the previous step, we obtain the optimal number of SUs (i.e., $\mathbf{a}^{*}=\left\{a_{1}^{*}, a_{2}^{*}, \cdots, a_{N}^{*}\right\}$ ) assigned to sense the PU channels. Note that the number of SUs assigned to sense a certain channel $n(n \in \mathcal{N})$ could be zero, i.e., that channel is determined not to be sensed. To address this problem, we introduce the following indicator function

$$
I\left(a_{n}^{*}\right)=\left\{\begin{array}{cc}
0 & \text { for } a_{n}^{*}=0, \\
1 & \text { otherwise }
\end{array}\right.
$$

Denote $U^{\prime}\left(X \mid \mathbf{a}^{*}\right)$ as the first term of utility function (10) in the case where $\mathbf{a}^{*}$ is given. Again we ignore the second term in (10) since it is a constant when $\kappa$ is fixed. $U^{\prime}\left(X \mid \mathbf{a}^{*}\right)$ has the following expression

$$
\begin{aligned}
& U^{\prime}\left(X \mid \mathbf{a}^{*}\right)=\sum_{n}\left[\alpha_{n} I\left(a_{n}^{*}\right) \prod_{m}\left(1-p_{f, m, n}\right)^{x_{m, n}}\right] \\
& =\sum_{n}\left[\alpha_{n} I\left(\sum_{m} x_{m, n}\right) e^{\sum_{m} \beta_{m, n} x_{m, n}}\right]=\sum_{n}\left[\alpha_{n} f_{n}\left(\mathbf{x}_{n}\right)\right],
\end{aligned}
$$

where $\beta_{m, n}=\ln \left(1-p_{f, m, n}\right), \quad f_{n}\left(\mathbf{x}_{n}\right)=$ $I\left(\sum_{m} x_{m, n}\right) e^{\sum_{m} \beta_{m, n} x_{m, n}}$ and $\sum_{m} x_{m, n}=a_{n}^{*}$. For the coefficient $\beta_{m, n}$, we give the following remark to describe its characteristic.

Remark 1. If the number of SUs assigned to sense each channel (i.e., $\mathbf{a}=\left\{a_{1}, a_{2}, \cdots, a_{N}\right\}$ ) is given, then $\beta_{m, n}$ is a precalculated constant $\forall m \in \mathcal{M}$ and $\forall n \in \mathcal{N}$.

The above remark can be explained as follows. According to equation $\beta_{m, n}=\ln \left(1-p_{f, m, n}\right)$, the value of $\beta_{m, n}$ is determined by $p_{f, m, n}$. For fixed $m \in \mathcal{M}, n \in \mathcal{N}$ and given system setting, the value of $p_{f, m, n}$ depends on $p_{d, n}$ only according to equation (4). Since $P_{d, n}$ is bounded to $\lambda_{n}$ according to Appendix A, we can conclude that $p_{d, n}$ is a precalculated constant if $a_{n}$ is fixed according to equation (3). Therefore, the coefficient $\beta_{m, n}$ is a precalculated constant when $\mathbf{a}=\left\{a_{1}, a_{2}, \cdots, a_{N}\right\}$ is given.

With the results obtained in the first step, the assignment subproblem is given as follows.

$$
\begin{aligned}
(\mathbf{C S S 2}): & \max _{X} U^{\prime}\left(X \mid \mathbf{a}^{*}\right) \\
\text { s.t. } & \sum_{m=1}^{M} x_{m, n}=a_{n}^{*}, \\
& (12)-(14) .
\end{aligned}
$$

In the remaining part of this subsection, subproblem (CSS2) will be analyzed in the framework of $L / L^{\natural}$-convexity. The following Proposition 2 states the supermodular property of function $f_{n}\left(\mathbf{x}_{n}\right)$.

Proposition 2. Function $f_{n}\left(\mathbf{x}_{n}\right)$ defined in (34) with its domain as $\operatorname{dom} f_{n} \subseteq\{0,1\}^{M}$ is a supermodular function.

Proof: Let $\mathbf{x}_{n}^{\prime}$ and $\mathbf{x}_{n}^{\prime \prime}$ be in $\operatorname{dom} f_{n}$ and

$$
\begin{aligned}
& f_{n}\left(\mathbf{x}_{n}^{\prime}\right)=I\left(\sum_{m} x_{m, n}^{\prime}\right) e^{\sum_{m} \beta_{m, n} x_{m, n}^{\prime}}, \\
& f_{n}\left(\mathbf{x}_{n}^{\prime \prime}\right)=I\left(\sum_{m} x_{m, n}^{\prime \prime}\right) e^{\sum_{m} \beta_{m, n} x_{m, n}^{\prime \prime}},
\end{aligned}
$$

then we have

$$
\begin{aligned}
& f_{n}\left(\mathbf{x}_{n}^{\prime}\right)+f_{n}\left(\mathbf{x}_{n}^{\prime \prime}\right)=I\left(\sum_{m} x_{m, n}^{\prime}\right) e^{\sum_{m} \beta_{m, n} x_{m, n}^{\prime}} \\
& +I\left(\sum_{m} x_{m, n}^{\prime \prime}\right) e^{\sum_{m} \beta_{m, n} x_{m, n}^{\prime \prime}}
\end{aligned}
$$

$$
\begin{aligned}
& f_{n}\left(\mathbf{x}_{n}^{\prime} \wedge \mathbf{x}_{n}^{\prime \prime}\right)+f_{n}\left(\mathbf{x}_{n}^{\prime} \vee \mathbf{x}_{n}^{\prime \prime}\right) \\
& =I\left(\sum_{m} \min \left\{x_{m, n}^{\prime}, x_{m, n}^{\prime \prime}\right\}\right) e^{\sum_{m} \beta_{m, n} \min \left\{x_{m, n}^{\prime}, x_{m, n}^{\prime \prime}\right\}} \\
& +I\left(\sum_{m} \max \left\{x_{m, n}^{\prime}, x_{m, n}^{\prime \prime}\right\}\right) e^{\sum_{m} \beta_{m, n} \max \left\{x_{m, n}^{\prime}, x_{m, n}^{\prime \prime}\right\}} .
\end{aligned}
$$

In the next part, we will prove this proposition in three cases:

Case 1: $\mathbf{x}_{n}^{\prime}=\mathbf{0}, \mathbf{x}_{n}^{\prime \prime}=\mathbf{0}$ or $\mathbf{x}_{n}^{\prime \prime} \neq \mathbf{0}$

If $\mathbf{x}_{n}^{\prime \prime}=\mathbf{0}$, then

$$
f_{n}\left(\mathbf{x}_{n}^{\prime}\right)+f_{n}\left(\mathbf{x}_{n}^{\prime \prime}\right)=f_{n}\left(\mathbf{x}_{n}^{\prime} \wedge \mathbf{x}_{n}^{\prime \prime}\right)+f_{n}\left(\mathbf{x}_{n}^{\prime} \vee \mathbf{x}_{n}^{\prime \prime}\right)=0 .
$$

If $\mathbf{x}_{n}^{\prime \prime} \neq \mathbf{0}$, then

$f_{n}\left(\mathbf{x}_{n}^{\prime}\right)+f_{n}\left(\mathbf{x}_{n}^{\prime \prime}\right)=f_{n}\left(\mathbf{x}_{n}^{\prime} \wedge \mathbf{x}_{n}^{\prime \prime}\right)+f_{n}\left(\mathbf{x}_{n}^{\prime} \vee \mathbf{x}_{n}^{\prime \prime}\right)=e^{\sum_{m} \beta_{m, n} x_{m, n}^{\prime \prime}}$.

Equations (40) and (41) indicate that $f_{n}\left(\mathbf{x}_{n}\right)$ is a supermodular function when $\mathbf{x}_{n}^{\prime}=\mathbf{0}$. In the following two cases, let $\mathbf{x}_{n}^{\prime} \neq \mathbf{0}$ and $\mathbf{x}_{n}^{\prime \prime} \neq \mathbf{0}$.

Case 2: $\mathbf{x}_{n}^{\prime} \neq \mathbf{0}, \mathbf{x}_{n}^{\prime \prime} \neq \mathbf{0}$ and $\mathbf{x}_{n}^{\prime}=\mathbf{x}_{n}^{\prime \prime}$

Here, we define vectors $\mathbf{x}_{n}^{\prime}$ and $\mathbf{x}_{n}^{\prime \prime}$ as equal (i.e., $\mathbf{x}_{n}^{\prime}=$ $\mathbf{x}_{n}^{\prime \prime}$ ) if every element in these two vectors is equal (i.e., $\left.x_{m, n}^{\prime}=x_{m, n}^{\prime \prime} \in\{0,1\}\right)$. Similarly, $\mathbf{x}_{n}^{\prime} \neq \mathbf{x}_{n}^{\prime \prime}$ if there exists $m \in \mathcal{M}$ such that $x_{m, n}^{\prime} \neq x_{m, n}^{\prime \prime} \in\{0,1\}$. In this case, $\min \left\{x_{m, n}^{\prime}, x_{m, n}^{\prime \prime}\right\}=\max \left\{x_{m, n}^{\prime}, x_{m, n}^{\prime \prime}\right\}=x_{m, n}^{\prime}=x_{m, n}^{\prime \prime}$. Therefore,

$$
f_{n}\left(\mathbf{x}_{n}^{\prime}\right)+f_{n}\left(\mathbf{x}_{n}^{\prime \prime}\right)=f_{n}\left(\mathbf{x}_{n}^{\prime} \wedge \mathbf{x}_{n}^{\prime \prime}\right)+f_{n}\left(\mathbf{x}_{n}^{\prime} \vee \mathbf{x}_{n}^{\prime \prime}\right) .
$$

Equation (42) indicates that $f_{n}\left(\mathbf{x}_{n}\right)$ is a supermodular function when $\mathbf{x}_{n}^{\prime}=\mathbf{x}_{n}^{\prime \prime}$.

Case $3: \mathbf{x}_{n}^{\prime} \neq \mathbf{0}, \mathbf{x}_{n}^{\prime \prime} \neq \mathbf{0}$ and $\mathbf{x}_{n}^{\prime} \neq \mathbf{x}_{n}^{\prime \prime}$

Denote $\mathcal{B}_{1}, \mathcal{B}_{0}, \mathcal{B}^{\prime}$ and $\mathcal{B}^{\prime \prime}$ as sets which satisfy the following 
conditions:

$\mathcal{B}_{1}=\left\{m \mid x_{m, n}^{\prime}=x_{m, n}^{\prime \prime}=1\right\}, \mathcal{B}_{0}=\left\{m \mid x_{m, n}^{\prime}=x_{m, n}^{\prime \prime}=0\right\}$,

$\mathcal{B}^{\prime}=\left\{m \mid x_{m, n}^{\prime}=1, x_{m, n}^{\prime \prime}=0\right\}, \mathcal{B}^{\prime \prime}=\left\{m \mid x_{m, n}^{\prime}=0, x_{m, n}^{\prime \prime}=1\right\}$.

It is not difficult to notice that

$$
\mathcal{B}_{1} \cup \mathcal{B}_{0} \cup \mathcal{B}^{\prime} \cup \mathcal{B}^{\prime \prime}=\mathcal{M} \text {. }
$$

We then obtain

$$
\begin{aligned}
& f_{n}\left(\mathbf{x}_{n}^{\prime}\right)+f_{n}\left(\mathbf{x}_{n}^{\prime \prime}\right) \\
& =e^{\sum_{m \in\left(\mathcal{B}^{\prime} \cup \mathcal{B}_{1}\right)} \beta_{m, n} x_{m, n}^{\prime}}+e^{\sum_{m \in\left(\mathcal{B}^{\prime \prime} \cup \mathcal{B}_{1}\right)} \beta_{m, n} x_{m, n}^{\prime \prime}} \\
& =e^{\sum_{m \in \mathcal{B}_{1}} \beta_{m, n}}\left(e^{\sum_{m \in \mathcal{B}^{\prime}} \beta_{m, n}}+e^{\sum_{m \in \mathcal{B}^{\prime \prime}} \beta_{m, n}}\right),
\end{aligned}
$$

and

$f_{n}\left(\mathbf{x}_{n}^{\prime} \wedge \mathbf{x}_{n}^{\prime \prime}\right)+f_{n}\left(\mathbf{x}_{n}^{\prime} \vee \mathbf{x}_{n}^{\prime \prime}\right)=e^{\sum_{m \in \mathcal{B}_{1}} \beta_{m, n}}+e^{\sum_{m \in \mathcal{M} / \mathcal{B}_{0}} \beta_{m, n}}$ $=e^{\sum_{m \in \mathcal{B}_{1}} \beta_{m, n}}\left(1+e^{\sum_{m \in \mathcal{M} / \mathcal{B}_{0} / \mathcal{B}_{1}} \beta_{m, n}}\right)$.

Observe that

$$
\begin{aligned}
& e^{\sum_{m \in \mathcal{B}^{\prime}} \beta_{m, n}}+e^{\sum_{m \in \mathcal{B}^{\prime \prime}} \beta_{m, n}}-e^{\sum_{m \in \mathcal{M} / \mathcal{B}_{0} / \mathcal{B}_{1}} \beta_{m, n}} \\
& =e^{\sum_{m \in \mathcal{B}^{\prime}} \beta_{m, n}}+e^{\sum_{m \in \mathcal{B}^{\prime \prime}} \beta_{m, n}}\left(1-e^{\sum_{m \in \mathcal{B}^{\prime}} \beta_{m, n}}\right) \\
& <e^{\sum_{m \in \mathcal{B}^{\prime}} \beta_{m, n}}+\left(1-e^{\sum_{m \in \mathcal{B}^{\prime}} \beta_{m, n}}\right)=1 .
\end{aligned}
$$

According to the deduction given above, we have the following inequality

$$
\begin{gathered}
e^{\sum_{m \in \mathcal{B}_{1}} \beta_{m, n}}\left(e^{\sum_{m \in \mathcal{B}^{\prime}} \beta_{m, n}}+e^{\sum_{m \in \mathcal{B}^{\prime \prime}} \beta_{m, n}}\right) \\
<e^{\sum_{m \in \mathcal{B}_{1}} \beta_{m, n}}\left(1+e^{\sum_{m \in \mathcal{M} / \mathcal{B}_{0} / \mathcal{B}_{1} \beta_{m, n}}}\right) .
\end{gathered}
$$

The above inequality (46) implies the following result

$$
f_{n}\left(\mathbf{x}_{n}^{\prime}\right)+f_{n}\left(\mathbf{x}_{n}^{\prime \prime}\right)<f_{n}\left(\mathbf{x}_{n}^{\prime} \wedge \mathbf{x}_{n}^{\prime \prime}\right)+f_{n}\left(\mathbf{x}_{n}^{\prime} \vee \mathbf{x}_{n}^{\prime \prime}\right) .
$$

Inequality (47) indicates that $f_{n}\left(\mathbf{x}_{n}\right)$ is a supermodular function when $\mathbf{x}_{n}^{\prime} \neq \mathbf{x}_{n}^{\prime \prime}$. This completes the proof.

With Proposition 2 and Corollary 2, we further state the inherent $\mathrm{L}^{\natural}$-concave property of function $f_{n}\left(\mathbf{x}_{n}\right)$ in the following theorem.

Theorem 4. Function $f_{n}\left(\mathbf{x}_{n}\right)$ with its domain as $\operatorname{dom} f_{n} \subseteq$ $\{0,1\}^{M}$ is an $L^{\natural}$-concave function.

Proof: Since the assignment matrix $X$ is binary, domain of $f_{n}\left(\mathbf{x}_{n}\right)$ is a subset of $\{0,1\}^{M}$. According to Proposition 2, $f_{n}\left(\mathbf{x}_{n}\right)$ is a supermodular function. Corollary 2 tells us that a function $f$ with $\operatorname{dom} f \subseteq\{0,1\}^{M}$ is $\mathrm{L}^{\natural}$-concave if and only if it is supermodular. Therefore, function $f_{n}\left(\mathbf{x}_{n}\right)$ is an $\mathrm{L}^{\natural}$-concave function.

Based on Theorems 2 and 4, we are ready to give the following Theorem 5, which is of profound importance in the analysis of subproblem (CSS2) and describes a very nice property to design an efficient algorithm.

Theorem 5. The objective function of subproblem (CSS2) $U^{\prime}\left(X \mid \mathbf{a}^{*}\right)$ is an $L^{\natural}$-concave function. Consequently, $U^{\prime}\left(X \mid \mathbf{a}^{*}\right)$ is an integrally concave function.

Proof: According to equation (34), $U^{\prime}\left(X \mid \mathbf{a}^{*}\right)$ is the sum of a group of $\mathrm{L}^{\natural}$-concave functions. Based on Theorem 2, the sum of $L^{\natural}$-convex functions are still $L^{\natural}$-convex function.
Algorithm 2 Steepest Descent algorithm to find the SU assignment matrix $X$ given $\mathbf{a}^{*}$

1: Input: The number of SUs participating in sensing $(M)$, the PU channel number $(N)$, the detection probability requirements $\left(\lambda_{1}, \lambda_{2}, \ldots, \lambda_{N}\right), \alpha_{n}=w_{r} P_{n}\left(\mathcal{H}_{0}\right) C_{0, n}$ and the SNR matrix $\Gamma=\left\{\gamma_{m, n}\right\}$.

2: Given $\mathbf{a}^{*}$, initialize $X$ as any feasible point and let $\mathbf{x}=$ $\left[\mathbf{x}_{1}^{T}, \mathbf{x}_{2}^{T}, \cdots, \mathbf{x}_{N}^{T}\right]$. Note that the dimension of $\mathbf{x}$ is $1 \times$ $M N$.

3: Find $u \in \operatorname{supp}^{+}(\mathbf{x})$ and $v \in \operatorname{supp}^{+}(\mathbf{1}-\mathbf{x})(u, v \in$ $\{1,2, \cdots, M N\})$ that make $\mathbf{x}-e_{u}+e_{v}$ feasible and maximize $U_{G}\left(\mathbf{x}-e_{u}+e_{v}\right)$, where $e_{u}$ and $e_{v}$ are unit vectors.

4: If $U_{G}\left(\mathbf{x}-e_{u}+e_{v}\right) \geq U_{G}(\mathbf{x})$, then set $\mathbf{x}:=\mathbf{x}-e_{u}+e_{v}$ and go to line 3.

5: Otherwise, output $X=\left[\mathbf{x}_{1}, \mathbf{x}_{2}, \ldots, \mathbf{x}_{N}\right]$ and stop.

Therefore, we can conclude that $U^{\prime}\left(X \mid \mathbf{a}^{*}\right)$ is an $\mathrm{L}^{\natural}$-concave function. Based on Lemma 1, we can conclude that $U^{\prime}\left(X \mid \mathbf{a}^{*}\right)$ is also an integrally concave function.

Theorem 5 states the $L^{\natural}$-concave and integrally concave properties of the objective function $U^{\prime}\left(X \mid \mathbf{a}^{*}\right)$ in subproblem (CSS2). Therefore, the local optimum of this function implies global optimum.

Similar to the proof in Appendix A, we can prove that constraint (14) should be bounded to get the optimal solution of subproblem (CSS2). We can always make the equality hold for constraint (14) by tuning the sensing threshold based on the relationship between $P_{d, n}$ and $P_{f, n}$. Therefore, the effective domain of the subproblem (CSS2) is a polyhedron which is restricted by constraints (36), (12) and (13). According to Theorem 5, the objective function of subproblem (CSS2) $U^{\prime}\left(X \mid \mathbf{a}^{*}\right)$ is an $\mathrm{L}^{\natural}$-concave function. This means that local optimality guarantees global optimality in an unconstrained optimization problem. Unfortunately, available local search method for integrally concave problem needs combinatorial computational complexity [21]. Hence, we propose to use the following Steepest Descent algorithm (Algorithm 2) to find the solution of subproblem (CSS2). Since only one dimensional search is done in the following Steepest Descent algorithm, the local optimality is not guaranteed.

Algorithm 2 initializes a feasible $X$ based on the given $\mathbf{a}^{*}$ in line 2. With the starting feasible $X$, a steepest descent searching procedure is executed from line 3 to line 5. Finally, the assignment matrix $X=\left[\mathbf{x}_{1}, \mathbf{x}_{2}, \ldots, \mathbf{x}_{N}\right]$ is outputted as the final result. The computational complexity of Algorithm 2 is $O\left(M^{2} N^{2}\right)$.

\section{Find the Optimal $\kappa$}

In this subsection, we will find a proper $\kappa$ that gives a good balance between spectrum sensing performance and sensing energy consumption. Let $X \mid a^{*}(\kappa)$ denote the SU assignment result obtained from Algorithm 2 when $\kappa$ SUs participate in sensing. We then define the marginal increase of utility 
function (10) after adding one more SU as follows

$$
\begin{aligned}
& \Delta U_{G}(\kappa) \triangleq\left[U^{\prime}\left(X \mid a^{*}(\kappa)\right)-w_{e} E(\kappa)\right]-\left[U^{\prime}\left(X \mid a^{*}(\kappa-1)\right)-\right. \\
& \left.w_{e} E(\kappa-1)\right]=\Delta U^{\prime}(\kappa)-\theta,
\end{aligned}
$$

where $\Delta U^{\prime}(\kappa)=U^{\prime}\left(X \mid a^{*}(\kappa)\right)-U^{\prime}\left(X \mid a^{*}(\kappa-1)\right)$ and $\theta=$ $w_{e} \tau\left(\phi_{s}-\phi_{e}\right)$. We then have the following observation.

Observation 1. The marginal increase $\Delta U^{\prime}(\kappa)$ is nonincreasing with respect to $\kappa$.

This observation will be validated later by simulations in section VI. The physical meaning of this observation is actually quite intuitive and we explain it through the following example. Consider a situation in which we first choose one SU, say $\mathrm{SU} i$, that gives the largest marginal increase in objective value $^{4}$ to perform sensing from $M$ available SUs. Later one more $\mathrm{SU}$, say $\mathrm{SU} j$, that gives the largest marginal increase is selected to participate in sensing from the remaining $M-1$ available SUs. The marginal increase given by SU $i$ must be larger than that of SU $j$. Otherwise, $\mathrm{SU} j$ will be chosen to sense first.

Based on Observation 1, we give the following proposition.

Proposition 3. The optimal number of SUs participating in sensing (i.e., $\left.\kappa^{*}\right)$ should satisfy the following conditions

$$
\Delta U^{\prime}\left(\kappa^{*}\right) \geq \theta \text { and } \Delta U^{\prime}\left(\kappa^{*}+1\right)<\theta .
$$

The physical meaning of Proposition 3 is as follows: the number of SUs participating in sensing should stop increasing when it yields negative marginal increase in utility function (10). We can use condition (49) as the testing criteria for $\kappa^{*}$ and this gives Algorithm 3.

In Algorithm 3, the complete SU assignment scheme is given by iteratively executing the three steps given above. Although the results obtained in Steps S1 and S3 are optimal, the complete SU assignment scheme is suboptimal because of the averaging of SNR in Step S1 and the non-optimality due to the local search in Step S2. The advantage of our proposed algorithm is summarized as follows. (1) Since the proposed algorithm is based on the $\mathrm{M} / \mathrm{M}^{\natural}$-convex and $\mathrm{L} / \mathrm{L}^{\natural}$-convex property of the problem, it is efficient and its computational complexity is low, i.e. $O\left(M^{3} N^{2}\right)$. (2) As shown later in the simulation results of Section VI, our proposed algorithm produces good SU assignment result which outperforms the best existing heuristic algorithm and the classical genetic algorithm. (3) Our proposed algorithm is applicable in a more practical situation where SUs individually have heterogeneous SNR for different PU channels and PU channels have heterogeneous detection probability requirement, channel capacity and stationary idle (busy) probability. (4) The algorithm can be easily extended to a new scenario where "AND" fusion rule is adopted or channel fading is considered directly.

\section{NUMERICAL RESULTS}

In this section, we provide several numerical examples to validate the theoretical analysis and evaluate the performance

\footnotetext{
${ }^{4}$ The term objective value is the value of the objective function for a given system setting and SUs assignment solution.
}

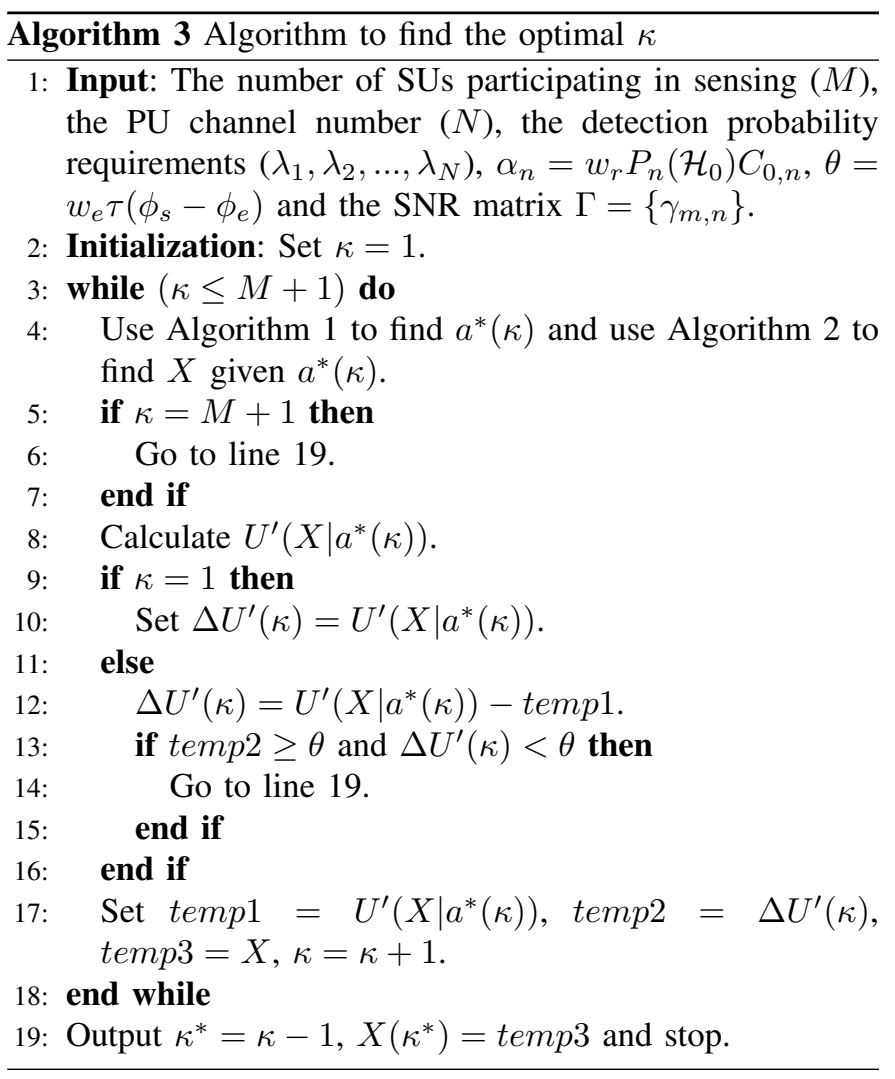

of our proposed SU assignment scheme. The parameter setting used in the simulation is as follows: the sampling rate $f_{s}=1 \mathrm{MHz}$, the sensing duration $\tau=50 \mathrm{~ms}$, total number of SUs $M=20$ and the PU channel number $N=3$. Detection probability requirements $\left\{\lambda_{n} \mid n=1,2,3\right\}$ and the coefficients $\left\{\alpha_{n} \mid n=1,2,3\right\}^{5}$ for these three PU channels are listed in the Table I. SNR values are given in Table II $^{6}$.

TABLE I

SIMULATION PARAMETER SETTING FOR $\lambda_{n}$ AND $\alpha_{n}$ FOR $n=1,2,3$

\begin{tabular}{|l|lll|}
\hline$n$ & 1 & 2 & 3 \\
\hline$\lambda_{n}$ & 0.80 & 0.90 & 0.92 \\
\hline$\alpha_{n}$ & 2.00 & 2.30 & 2.50 \\
\hline
\end{tabular}

In Fig. 3, the obtained SU assignment result for SNR setting 1 is shown. In this SU assignment result, five SUs (SU 1, 3, 7,13 and 18) are assigned to sense channel 1, seven (SU 6, $8,9,12,17,19$ and 20) are assigned to sense channel 2 and eight (SU 2, 4, 5, 10, 11, 14,15 and 16) are assigned to sense channel 3.

In Figs. 5 and 6, we compare the performance of our proposed SU assignment scheme with the existing Maximum Weighted Sum Channel Gain (MWSCG) assignment scheme [18] and classical genetic algorithm [27]. As mentioned in

${ }^{5}$ For given weighting factor $w_{r}$, larger value of $\alpha_{n}$ represents more desirable or better static characteristic (stationary idle probability and channel capacity) of the primary channel.

${ }^{6}$ The appending $1(0)$ represents that the corresponding SU is assigned (not assigned) to sense that PU channel for given SNR setting 1 when all the 20 SUs participate in sensing. 
TABLE II

SIMULATION PARAMETER SETTING FOR $\gamma_{m, n}$

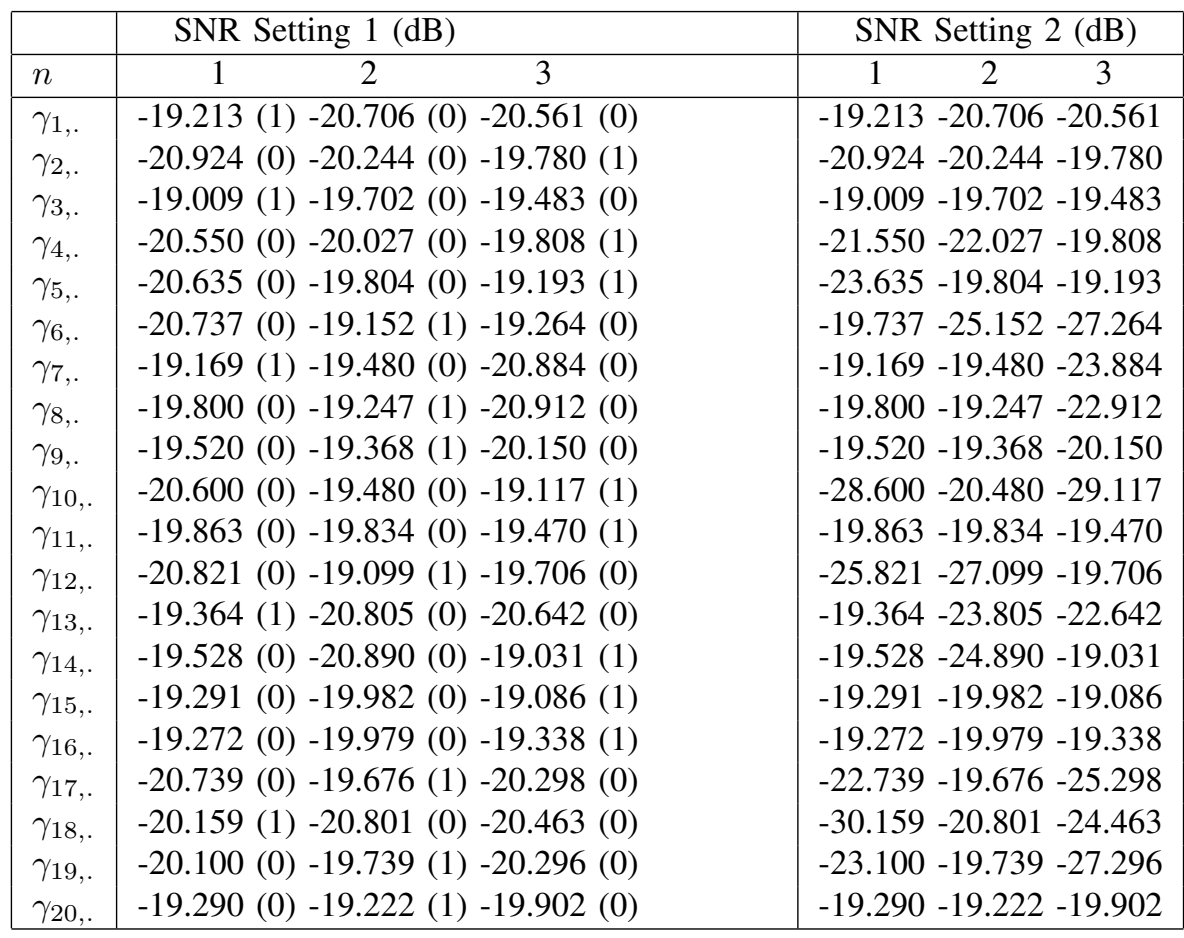

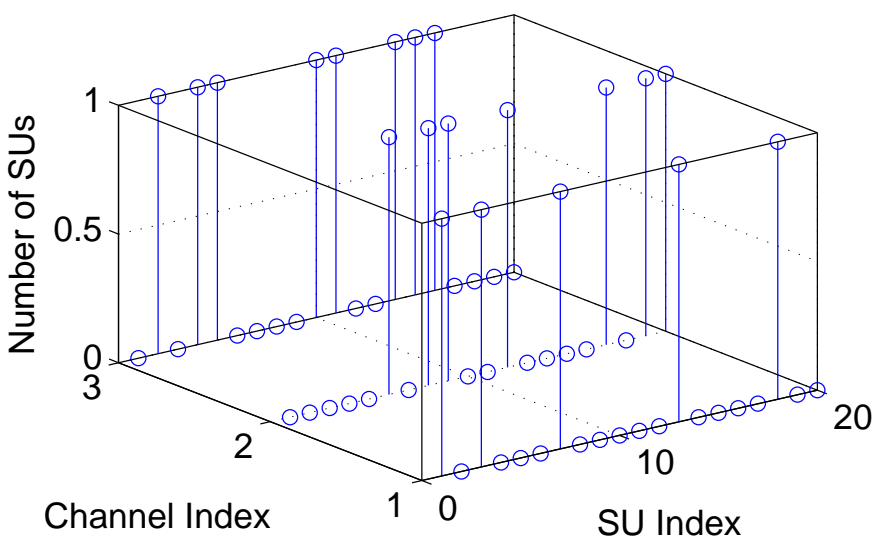

Fig. 3. SU assignment for given system parameters and SNR setting 1

[20], genetic algorithm is commonly used to solve multiobjective optimization problem. Hence, we choose it as a comparison benchmark. In our implementation of genetic algorithm, the initial population is 64 , the pairing method is Roulette wheel weighting and the mutation is single point. The MWSCG assignment method is the latest and best existing method to solve the general CSS problem to the best of our knowledge. For SNR setting 1, the proposed Steepest Descent algorithm outperforms the MWSCG assignment method and genetic algorithm as shown in Fig. 5. Fig. 6 shows that our proposed Steepest Descent algorithm performs better than the existing MWSCG assignment method and genetic algorithm for SNR setting 2 as well. The assignment of available SUs in

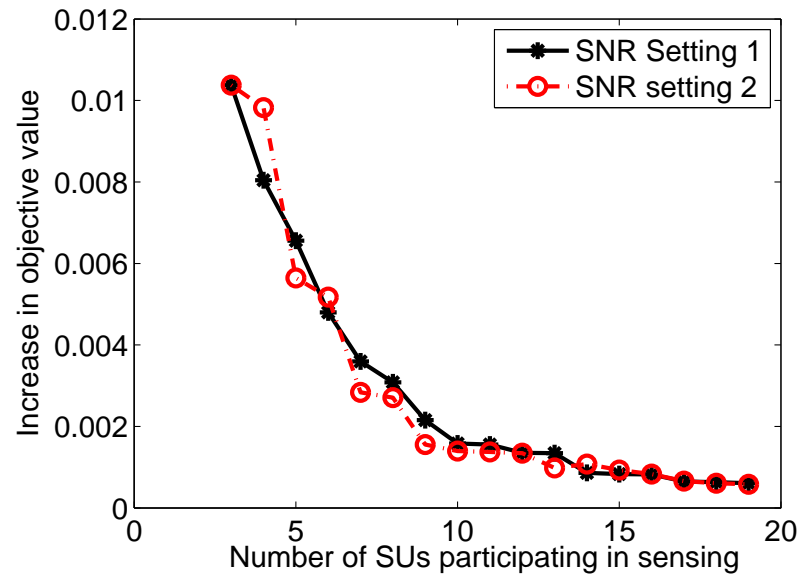

Fig. 4. Marginal increase of objective value with respect to $\kappa$

SNR setting 2 needs to be carefully dealt with, because several SUs (e.g., SU 6, 10, 12, 18 and 19) only have good SNR for a certain channel. The existing MWSCG assignment method has poor performance in this case. The MWSCG assignment scheme artificially lets the available SUs spread evenly to sense all PU channels and this makes the assignment method less effective.

Fig. 4 is given to verify Observation 1 . The marginal increase of objective value is non-increasing for both SNR setting 1 and SNR setting 2 as shown in Fig. 4. This simulation results agree with our analysis in subsection V-C. 


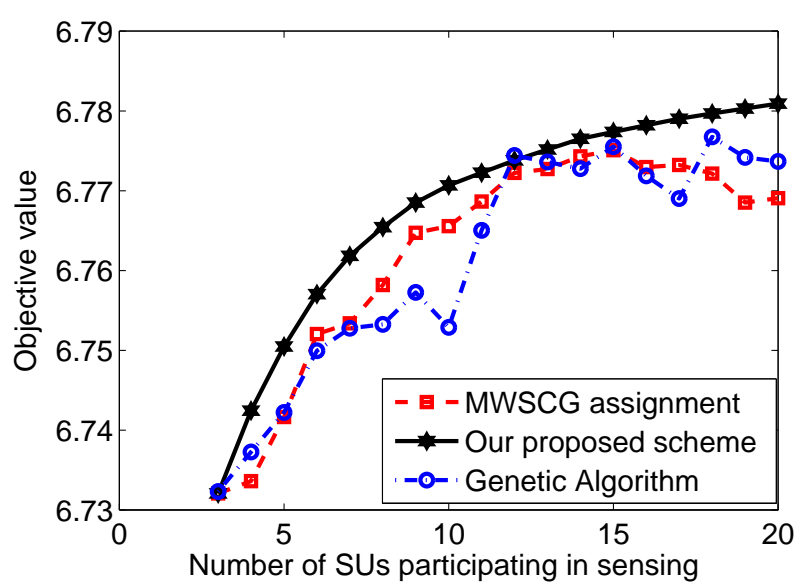

Fig. 5. Performance comparison between the existing MWSCG assignment method and our proposed Steepest Descent algorithm for SNR setting 1

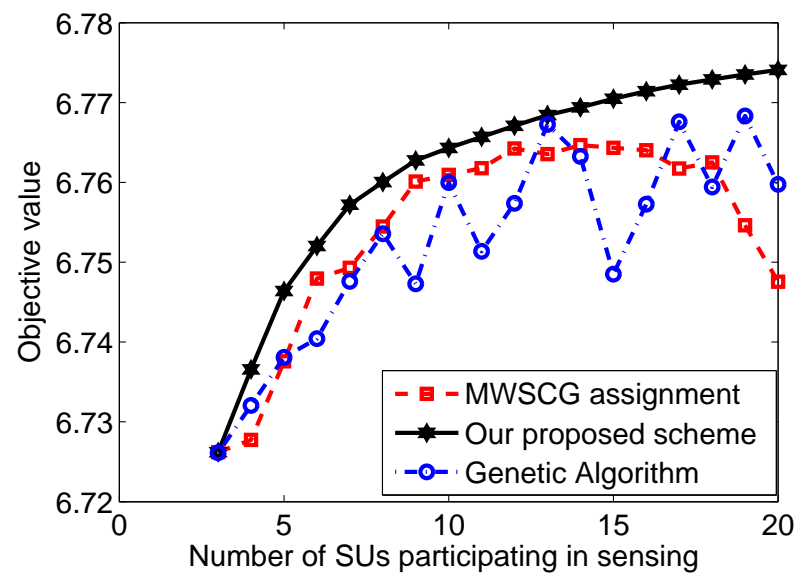

Fig. 6. Performance comparison between the existing MWSCG assignment method and our proposed Steepest Descent algorithm for SNR setting 2

\section{CONCLUSION}

In this paper, the CSS problem is studied in a practical scenario where (i) SUs individually have heterogeneous SNR for different PU channels and (ii) PU channels have heterogeneous detection probability requirement, channel capacity and stationary idle (busy) probability. Unlike many existing works that merely claim that this problem is NP hard and then turn to heuristic methods, we investigate the CSS problem in a solid discrete-convex framework. By taking both sensing performance and energy efficiency into consideration, we formulate the CSS problem as a nonlinear binary programming problem. A three-step approach is adopted to solve it. In Step $\mathrm{S} 1$, we analyze the CSS problem with the $\mathrm{M} / \mathrm{M}^{\natural}$-convex theory to determine the optimal number of SUs assigned to sense each channel. In Step S2, based on the results obtained in the previous step, we investigate the SU assignment scheme using the $\mathrm{L} / \mathrm{L}^{\natural}$-convex theory. In the last step, the optimal number of SUs participating in sensing is found based on the SU assignment obtained in Step S2. By combining these three steps, a complete and efficient SUs assignment scheme is obtained. Although the results obtained in Steps S1 and S3 are optimal, the complete SU assignment scheme is suboptimal because of the averaging of SNR in Step S1 and the non-optimality due to the local search in Step S2. Since our proposed scheme relies heavily on theoretical analysis, it outperforms the best existing method. We also provide numerical results to verify the theoretical analysis and evaluate the performance of our proposed SU assignment scheme.

\section{APPENDIX A}

\section{PROVE THAT CONSTRAINT (27) SHOULD BE BOUNDED TO} EQUALITY FOR SUBPROBLEM (CSS1)

If $a_{n}=0$, the detection requirement is meaningless since the channel is not sensed and thus will not be used by SUs. Therefore, we only need to prove for channels which are sensed by SUs (i.e., channels with $a_{n} \geq 1$ ) and constraint (27) should be bounded to equality for subproblem (CSS1). In this case, we have $\bar{P}_{f, n}\left(a_{n}\right)=P_{f, n}\left(a_{n}\right)$. Let $P_{d, n}=\lambda_{n}$ and $P_{d, n}^{\prime}>\lambda_{n}$, then the corresponding $P_{f, n}$ and $P_{f, n}^{\prime}$ can be expressed as follows.

$$
\begin{aligned}
& P_{f, n}=1-\left\{1-Q\left\{\varphi_{n} Q^{-1}\left[1-\left(1-P_{d, n}\right)^{1 / a_{n}}\right]+\phi_{n}\right\}\right\}^{a_{n}}, \\
& P_{f, n}^{\prime}=1-\left\{1-Q\left\{\varphi_{n} Q^{-1}\left[1-\left(1-P_{d, n}^{\prime}\right)^{1 / a_{n}}\right]+\phi_{n}\right\}\right\}^{a_{n}},
\end{aligned}
$$

where $\varphi_{n}=\sqrt{2 \bar{\gamma}_{n}+1}$ and $\phi_{n}=\sqrt{\tau f_{s}} \bar{\gamma}_{n}$. Denote $\nabla_{x} f$ as the first order differentiation of a function $f$ with respect to its argument $x$. Take the first order derivative of $P_{f, n}$ with respect to $P_{d, n}$ and we obtain

$$
\begin{aligned}
& \nabla_{P_{d, n}} P_{f, n}=\left\{1-Q\left[\varphi_{n} Q^{-1}\left(p_{d, n}\right)+\rho_{n}\right]\right\}^{a_{n}-1} \times \\
& \left(1-P_{d, n}\right)^{\frac{1-a_{n}}{a_{n}}} \times \exp \left\{\frac{\left[Q^{-1}\left(p_{d, n}\right)\right]^{2}-\left[\varphi_{n} Q^{-1}\left(p_{d, n}\right)+\phi_{n}\right]^{2}}{2}\right\}
\end{aligned}
$$

where $p_{d, n}=1-\left(1-P_{d, n}\right)^{\frac{1}{a_{n}}}$. Since $0<1-P_{d, n} \leq 1$ and $0<1-Q\left[\varphi_{n} Q^{-1}\left(p_{d}^{n}\right)+\phi_{n}\right]<1$, it is obvious that $\nabla_{P_{d, n}} P_{f, n}>0$. Therefore, $P_{f, n}$ is monotonically increasing with $P_{d, n}$. Since $P_{d, n}^{\prime}>P_{d, n}$, we have

$$
1-P_{f, n}^{\prime}<1-P_{f, n}
$$

Therefore, we have

$$
U^{\prime}(\mathbf{a})<U(\mathbf{a})
$$

where $U^{\prime}(\mathbf{a})=\sum_{n} \alpha_{n}\left[1-P_{f, n}^{\prime}\left(a_{n}\right)\right]$ and $U(\mathbf{a})=$ $\sum_{n} \alpha_{n}\left[1-P_{f, n}\left(a_{n}\right)\right]$. Therefore, the unbounded detection probability $P_{d, n}^{\prime}$ gives a worse objective value for subproblem (CSS1) compared with the bounded detection probability $P_{d, n}$. Hence, constraint (27) should be bounded to equality to achieve the optimal solution for subproblem (CSS1).

\section{APPENDIX B}

PROVE THAT CONSTRAINT (26) GIVES A POLYMATROID IN SUBPROBLEM (CSS1)

Let $F=M$ and $\mathbf{g}(\mathcal{N})=\sum_{n \in \mathcal{N}} a_{n}$, then constraint (26) equals to $G=\mathcal{G}(\mathcal{N}, F)=\left\{\mathbf{g} \in \mathbf{N}^{V}: \mathbf{g}(\mathcal{N}) \leq F(S)\right\}$. It is obvious that $F=M$ satisfies the following conditions:

(1) $F$ is nonnegative: $F(S) \geq 0$,

(2) $F$ is nondecreasing: $F(S) \leq F(T)$ if $S \subseteq T \subseteq V$, 
(3) $F$ is submodular: $F(S)+F(T) \geq F(S \cup T)+F(S \cap T)$. Therefore, $G=\mathcal{G}(\mathcal{N}, F)=\left\{\mathbf{g} \in \mathbf{N}^{V}: \mathbf{g}(\mathcal{N}) \leq F(S)\right\}$ is a polymatroid according to Definition 8 . Hence, constraint (26) gives a polymatroid in subproblem (CSS1). This completes the proof.

\section{REFERENCES}

[1] K. G. Shin, H. Kim, A. W. Min and A. Kumar,"Cognitive Radios for Dynamic Spectrum Access: from Concept to Reality," IEEE Wireless Commun. Mag., vol. 17, no. 6, pp. 64-74, Dec. 2010.

[2] Y. Zou, Y. Yao and B. Zheng, "Cooperative Relay Techniques for Cognitive Radio Systems: Spectrum Sensing and Secondary User Transmissions," IEEE Communications Magazine, pp. 98-103, Apr. 2012.

[3] K. B. Letaief and W. Zhang, "Cooperative Spectrum Sensing," Cognitive Wireless Communication Networks, Springer, pp. 115-138, Oct. 2007.

[4] D. Raychaudhuri and N. B. Mandayam, "Frontiers of Wireless and Mobile Communications," Proceedings of the IEEE, vol. 100, no. 4, pp. 824-840, Apr. 2012.

[5] M. Song, C. Xin, Y. Zhao and X. Cheng, "Dynamic Spectrum Access: from Cognitive Radio to Network Radio," IEEE Wireless Commun., vol. 19, no. 1, pp. 23-29, Feb. 2012.

[6] E. Z. Tragos, S. Zeadally, G. Fragkiadakis and V. A. Siris, "Spectrum Assignment in Cognitive Radio Networks: A Comprehensive Survey," to appear in IEEE Commun. Survey \& Tutorials, Jan. 9, 2013.

[7] R. Fan, H. Jiang, Q. Guo and Z. Zhang, "Joint Optimal Cooperative Sensing and Resource Allocation in Multichannel Cognitive Radio Networks," IEEE Trans. Veh. Technol., vol. 60, no. 2, pp. 722-729, Feb. 2011.

[8] R. Fan and H. Jiang, "Optimal Multi-Channel Cooperative Sensing in Cognitive Radio Networks," IEEE Trans. Wireless Commun.,vol. 9, no. 3, pp. 1128-1138, Mar. 2010

[9] E.C.Y. Peh, Y.C. Liang, Y.L. Guan and Y. Zeng, "Optimization of Cooperative Sensing in Cognitive Radio Networks: A Sensing-Throughput Tradeoff View," IEEE Trans. Vehicular Technology, vol. 58, no. 9, pp. 5294-5299, Nov. 2009

[10] Y.C. Liang, Y. Zeng, E.C.Y. Peh and A.T. Hoang, "Sensing-Throughput Tradeoff for Cognitive Radio Networks," IEEE Trans. Wireless Commun., vol. 7, no. 4, pp. 1326-1337, Apr. 2008.

[11] C. Song and Q. Zhang, "Cooperative Spectrum Sensing with MultiChannel Coordination in Cognitive Radio Networks," in Proc. of IEEE ICC 2010, pp. 1-5, Jul. 2010.

[12] T. Zhang and D.H.K. Tsang, "Optimal Cooperative Sensing Scheduling for Energy-Efficient Cognitive Radio Networks", IEEE INFOCOM 2011, pp. 2723-2731, Apr. 2011

[13] T. Zhang and D.H.K. Tsang, "Cooperative Sensing Scheduling for Energy-Aware Cognitive Radio Networks," Proc. of IEEE ICC 2011, pp. 1-6, Jul. 2011

[14] X. Sun, T. Zhang and D.H.K. Tsang, "Optimal Energy-Efficient Cooperative Sensing Scheduling for Cognitive Radio Networks with QoS Guarantee," Proc. of IEEE IWCMC 2011, pp. 1825-1830, Jul. 2011.

[15] X. Sun, L. Chen and D.H.K. Tsang, "Energy-efficient cooperative sensing scheduling for heterogeneous channel access in Cognitive Radio," Proc. of IEEE INFOCOM WKSHPS 2012, pp. 145-150, Mar. 2012.

[16] Y. Zou, Y. Yao and B. Zheng, "Outage Probability Analysis of Cognitive Transmissions: Impact of Spectrum Sensing Overhead", IEEE Trans. Wireless Commun., vol. 9, no. 8, pp. 2676-2688, Aug. 2010.

[17] Y. Zou, Y. Yao and B. Zheng, "A selective-relay based cooperative spectrum sensing scheme without dedicated reporting channels in cognitive radio networks", IEEE Trans. Wireless Commun., vol. 10, no. 4, pp. 1188-1198, Apr. 2011.

[18] P. Kaligineedi and V. K. Bhargava, "Sensor Allocation and Quantization Schemes for Multi-Band Cognitive Radio Cooperative Sensing System," IEEE Trans. Wireless Commun., vol. 10, no. 1, pp. 284-293, Jan. 2011.

[19] W. Zhang, R. K. Mallik and K. B. Letaief, "Optimization of cooperative spectrum sensing with energy detection in cognitive radio networks," IEEE Trans. Wireless Commun., vol. 8, no. 12, pp. 5761-5766, Dec. 2009.

[20] R.T. Marler and J.S. Arora, "Survey of multi-objective optimization methods for engineering," Structural and Multidisciplinary Optimization, vol. 26, no. 6, pp. 369-395, 2004.

[21] K. Murota, Discrete Convex Analysis, SIAM Monographs on Discrete Mathematics and Applications, pp. 133-203, 2003.
[22] A. Federgrue and H. Groenevel, "The Greedy Procedure for Resource Allocation Problems: Necessary and Sufficient Conditions for Optimality," Operations Research, vol. 34, no. 6, pp. 909-918, Nov.- Dec. 1986.

[23] G. Song and Y. Li, "Cross-Layer Optimization for OFDM Wireless Networks-Part II: Algorithm Development," IEEE Trans. Wireless Commun., vol. 4, no. 2, pp. 625-634, Mar. 2005.

[24] K. Murota and A. Shioura, "Relationship of M-/L-convex Functions with Discrete Convex Functions by Miller and Favati-Tardella," Discrete Applied Mathematics, vol. 115, pp. 15-176, 2001

[25] FCC, "Second Memorandum Opinion and Order in the Matter of Unlicensed Operation in the TV Broadcast Bands, Additional Spectrum for Unlicensed Devices Below $900 \mathrm{MHz}$ and in the $3 \mathrm{GHz}$ Band," Document 10-174, Sept. 23, 2010.

[26] ECC, Draft ECC Report 159, "Technical and Operational Requirements for the Possible Operation of Cognitive Radio Systems in the White Spaces of the Frequency Band 470-790 MHz", Sept. 2010

[27] R. L. Haupt and S. E. Haupt, "Practical Genetic Algorithms," Wileyinterscience, pp. 27-184, 2004.

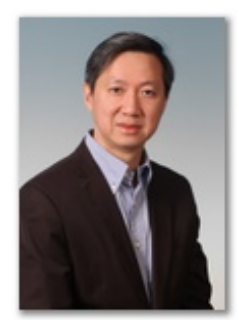

Danny H.K. Tsang (M'82-SM'00-F'12) received the Ph.D. degree in electrical engineering from the Moore School of Electrical Engineering at the University of Pennsylvania, U.S.A., in 1989. He has joined the Department of Electronic \& Computer Engineering at the Hong Kong University of Science and Technology since summer of 1992 and is now a professor in the department. He was a Guest Editor for the IEEE Journal of Selected Areas in Communications' special issue on Advances in P2P Streaming Systems, an Associate Editor for the Journal of Optical Networking published by the Optical Society of America, and a Guest Editor for the IEEE Systems Journal. He currently serves as Technical Editor for the IEEE Communications Magazine. He was nominated to become an IEEE Fellow in 2012. During his leave from HKUST in 20002001, Dr. Tsang assumed the role of Principal Architect at Sycamore Networks in the United States. He was responsible for the network architecture design of Ethernet MAN/WAN over SONET/DWDM networks. He also contributed to the $64 \mathrm{~B} / 65 \mathrm{~B}$ encoding proposal for Transparent GFP in the T1X1.5 standard which was advanced to become the ITU G.GFP standard. The coding scheme has now been adopted by International Telecommunication Union (ITU)'s Generic Framing Procedure recommendation GFP-T (ITU-T G.7041/Y.1303)). His current research interests include Internet quality of service, P2P video streaming, cloud computing, cognitive radio networks and smart grids.

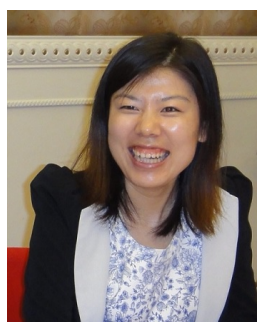

Xiangxia Sun received the B.E. degree in Communication Engineering from Harbin Institute of Technology, China, in 2010. She completed her M.phil degree from Hong Kong University of Science and Technology in 2012. During the M.phil study, her research interest was mainly on discrete-convex optimization and cognitive radio networks. She is currently employed in Societe Generale Corporate \& Investment Bank as an application support analyst. 